\title{
Étude multifactorielle de la coagulation mixte du lait analysée par viscoélasticimétrie
}

\author{
Y Noël 1, C Durier 2, N Lehembre 3, A Kobilinsky 2 \\ 1 INRA, station de recherches en technologie et analyses laitières, BP 89, 39800 Poligny \\ 2 INRA, laboratoire de biométrie, 78026 Versailles cédex; \\ 3 INRA, laboratoire de génie des procédés alimentaires, \\ 25, rue de la République, 91300 Massy, France
}

(Reçu le 23 avril 1990; accepté le 23 novembre 1990)

\begin{abstract}
Résumé - L'influence de la température, du pH d'emprésurage et de la dose de présure sur les cinétiques de coagulation mixte (présure et lactique) est étudiée dans le cadre d'un plan d'expérience multifactoriel. Les grandeurs caractéristiques des cinétiques de coagulation (temps, contrainte et angle de perte au point de latence, au point maximal et au point minimal, vitesse de raffermissement) sont mesurées avec un viscoélasticimètre. On montre que sur une faible étendue de variation $\left(32-34^{\circ} \mathrm{C}\right)$, la température a peu d'effet significatif sur ces grandeurs caractéristiques; les doses importantes de présure ont un effet limitant significatif sur le temps de latence et la contrainte maximale; le pH d'emprésurage joue un rôle important sur toutes les grandeurs caractéristiques. Un effet d'interaction est mis en évidence entre le $\mathrm{pH}$ d'emprésurage et la dose de présure. Des modèles de surfaces de réponse, construits avec la technique des polynômes orthogonaux et exprimant les grandeurs caractéristiques des cinétiques en fonction des facteurs étudiés, sont proposés.
\end{abstract}

coagulation mixte / étude multifactorielle / viscoélasticimétrie / température / $\mathrm{pH}$ d'emprésurage / dose de présure / polynôme orthogonal / surface de réponse

Summary - Multifactorial study of combined enzymatic and lactic milk coagulation measured by viscoelasticimetry. The influence of temperature, renneting $\mathrm{pH}$ and rennet quantity has been studied on combined enzymatic and lactic milk coagulation by multifactorial experimental design. Characteristic parameters of coagulation kinetics (time, stress, lag phase of lag point, maximum point, minimum point, maximum rate of firmness) were measured by a viscoelasticimetric method. Over a limited range of temperature variation $\left(32-34^{\circ} \mathrm{C}\right)$, temperature effect on kinetics has little significance; the high quantity of rennet may have a limiting effect on the lag time and maximum stress; renneting $\mathrm{pH}$ has an important effect on all the parameters. An interaction effect appears between renneting $\mathrm{pH}$ and quantity of rennet. Response surface model built on orthogonal polynomials are proposed to express the characteristic parameters of coagulation kinetics as functions of the factors studied.

combined enzymatic and lactic coagulation / multifactorial study / viscoelasticimetry / temperature / renneting $\mathrm{pH} /$ rennet quantity / orthogonal polynomial / response surface 


\section{INTRODUCTION}

Contrairement à la coagulation enzymatique, la coagulation mixte a été peu étudiée jusqu'ici (Van Hooydonk et al, 1986; Noël, 1989), la difficulté principale étant due à la maîtrise imparfaite de la croissance des bactéries lactiques. Or, une meilleure connaissance des phénomènes associés à la coagulation mixte, en plus de l'intérêt directement lié à la pratique fromagère, ouvre de nouvelles perspectives d'investigation des mécanismes de la coagulation du lait.

Par ailleurs, le développement des méthodes viscoélasticimétriques (Bohlin et al, 1984; Lee, 1986; Dejmek, 1987) autorise un suivi des cinétiques de coagulation dans des conditions peu perturbatrices permettant de caractériser le coagulum en terme de fermeté et de viscoélasticité.

Les facteurs généralement étudiés sont, d'une part, les facteurs de composition du lait, d'autre part le $\mathrm{pH}$, la température, la dose d'agent coagulant et la quantité de calcium ajouté. Pour cette première étude, nous n'avons pas retenu les facteurs de composition, et l'influence du calcium a été étudiée séparément (Noël, 1989). Le plan d'expérience, du type composite centré, a été construit sur 3 facteurs, la température, le $\mathrm{pH}$ à l'emprésurage et la dose d'enzyme.

L'influence de la température sur la coagulation du lait a été étudiée par différents auteurs, mais l'étendue explorée couvre le plus souvent 20-30 degrés (Kowalchyk et Olson, 1977; Ramet et Weber, 1980; Tokita et al, 1982, 1983; McMahon et Brown, 1984; Walstra et Jenness, 1984; Walstra et Van Vliet, 1986; Lee, 1986; Zoon et al, 1988b; Van Vliet et al, 1989).

On a choisi d'examiner ici l'influence de la température sur une faible plage de variation, $32-34{ }^{\circ} \mathrm{C}$, situation qui correspond à la pratique fromagère.
Dans la littérature, les effets de la dose de présure sont examinés sur des cinétiques de coagulation enzymatique pour des étendues de variation très diverses : le rapport entre la dose maximale et la dose minimale varie de 1,3 (Bolhin, 1984) à 80 (McMahon et Brown, 1984) en passant par 5 (Ramet et Weber, 1980; Zoon et al, 1988a) et 13 (Tokita et al, 1982).

L'influence de la dose de présure est examinée ici sur une plage de variation de 1 à 50 , ce qui recouvre les doses employées pour les diverses technologies fromagères (Eck, 1984).

Les travaux portant sur l'influence du $\mathrm{pH}$ sur la coagulation du lait concernent le plus souvent des gels enzymatiques acidifiés par voie chimique, jusqu'à un pH qui reste constant pendant la coagulation (Cheryan et al, 1975; Ramet et Weber, 1980; Shalabi et Fox, 1982; Van Hooydonk et al, 1986; Kim et Kinsella, 1989; Zoon et al, 1989). Harwalkar et Kalab (1981), ainsi que Roefs (1985), ont étudié la coagulation acide de gels obtenus par acidification chimique à $\mathrm{pH} \mathrm{4,6}$ et à froid, suivie d'un chauffage progressif. II existe quelques études de la coagulation du lait en cours d'acidification, soit chimique, notamment par la glucono-lactone (Harwalkar et Kalab, 1980; Heertje et al, 1985; Roefs, 1985 ), soit plus rarement lactique (Van Hooydonk et Van den Berg, 1988; Famelart et Maubois, 1988). Roefs (1985) a aussi étudié le comportement de gels obtenus par action de la présure sur du lait préalablement acidifié à froid entre $\mathrm{pH} 4,8$ et 5,8 puis réchauffé.

La plage de variation du $\mathrm{pH}$ à l'emprésurage a été choisie en tenant compte à la fois de la réalité de la pratique fromagère, et des caractéristiques (équidistance et centrage) que les niveaux devaient présenter dans le plan d'expérience.

Cette approche multifactorielle encore assez peu utilisée (Lacroix et Castaigne, 
1986) permet d'évaluer non seulement les effets principaux des facteurs mais aussi leurs interactions. Enfin, pour limiter les paramètres, une seule souche de bactéries lactiques a été utilisée.

\section{MATÉRIELS ET MÉTHODES}

\section{Le lait}

Le lait est reconstitué à partir d'une poudre de lait écrémé Nilac (NIZO, Pays-Bas) du type basse température, sur la base de $100 \mathrm{~g}$ de poudre et $1 \mathrm{mmol}^{\circ-1} \mathrm{CaCl}_{2}$ par $\mathrm{kg}$ de lait selon le protocole décrit précédemment (Noël, 1989).

\section{La solution enzymatique}

La solution enzymatique est préparée avec une présure en poudre purifiée (Hansen, Hala). Celle-ci contient $4438 \mathrm{mg}$ de chymosine active et $63 \mathrm{mg}$ de pepsine bovine par $\mathrm{kg}$ de poudre déterminés selon la norme FIL $110 \mathrm{~A}: 1987$. La présure est mise en solution à la concentration de $8 \%(\mathrm{~m} / \mathrm{v})$ dans un tampon acétate de sodium $0,05 \mathrm{~mol}^{-1} \mathrm{I}^{-1}, \mathrm{pH} 5,5$, contenant $0,1 \%(\mathrm{~m} / \mathrm{v})$ de sérum albumine utilisée pour ses propriétés stabilisantes. La solution est fractionnée dans des tubes à hémolyse avant d'être congelée. Au moment de son utilisation, la solution enzymatique est décongelée, éventuellement diluée pour obtenir les concentrations requises. La solution enzymatique est ajoutée à raison de $0,5 \mathrm{ml}$ par $\mathrm{kg}$ de lait.

\section{Les ferments lactiques}

Une seule souche lactique a été utilisée : Streptococcus lactis diacetylactis (CNRZ 125). Elle est conservée et fournie sous forme de culture concentrée lyophilisée. Le choix de cette souche a été déterminé en raison de sa très bonne activité acidifiante et de sa régularité, estimée par la valeur moyenne de la production d'acide lactique calculée sur 5 années : $49,3^{\circ} \mathrm{D}$ (écart type $=4,6$ ).
La souche lyophilisée est remise en suspension dans $10 \mathrm{ml}$ de lait stérilisé, puis incubée à $25{ }^{\circ} \mathrm{C}$ jusqu'à coagulation du lait. La souche dans le milieu coagulé est repiquée à $1 \%$ dans $10 \mathrm{ml}$ de lait stérilisé. Les repiquages sont renouvelés toutes les $24 \mathrm{~h}$ jusqu'à l'obtention d'une souche active. L'activité acidifiante est contrôlée par la mesure de la quantité d'acide lactique produit (exprimée en ${ }^{\circ} \mathrm{D}$ selon la méthode INRA) par la souche incubée à $30^{\circ} \mathrm{C}$. Lorsque l'activité est stable, ce qui nécessite 56 repiquages, des tubes de $5 \mathrm{ml}$ de lait stérilisé sont ensemencés à $5 \%$ avec le dernier repiquage. Ces tubes sont congelés rapidement dans un bain d'alcool placé dans un congélateur à $-30^{\circ} \mathrm{C}$, puis stockés dans celui-ci. La souche congelée est remise en activité la semaine précédant les essais.

\section{Les mesures rhéologiques}

Les mesures rhéologiques ont été réalisées comme décrit précédemment (Noël, 1989) avec le viscoélasticimètre GVT-INRA (Gervais et al, 1982).

\section{Cinétique de coagulation mixte}

La cinétique de coagulation mixte a été décrite précédemment (Noël, 1989). On a choisi de représenter sur la figure 1 plusieurs allures de cinétiques pour illustrer leur diversité : chaque graphe indique l'évolution de la contrainte, de l'angle de perte et du $\mathrm{pH}$. L'addition des ferments lactiques est effectuée au temps 0 , celle de l'enzyme coagulante au temps $t_{0}$ matérialisé par une flèche sur chacun des graphiques de la figure 1. Les points caractéristiques, utilisés pour l'analyse des courbes, sont les suivants :

- le point $P_{L}$ qui correspond à la fin du temps de latence (les valeurs du temps de latence sont calculées à partir de $t_{0}$ ),

- le point $P_{M}$ qui correspond au maximum de la contrainte,

- le point $P_{m}$ qui correspond au minimum de la contrainte,

- le point $P_{6}$ qui correspond à $6 \mathrm{~h}$ après l'emprésurage. 

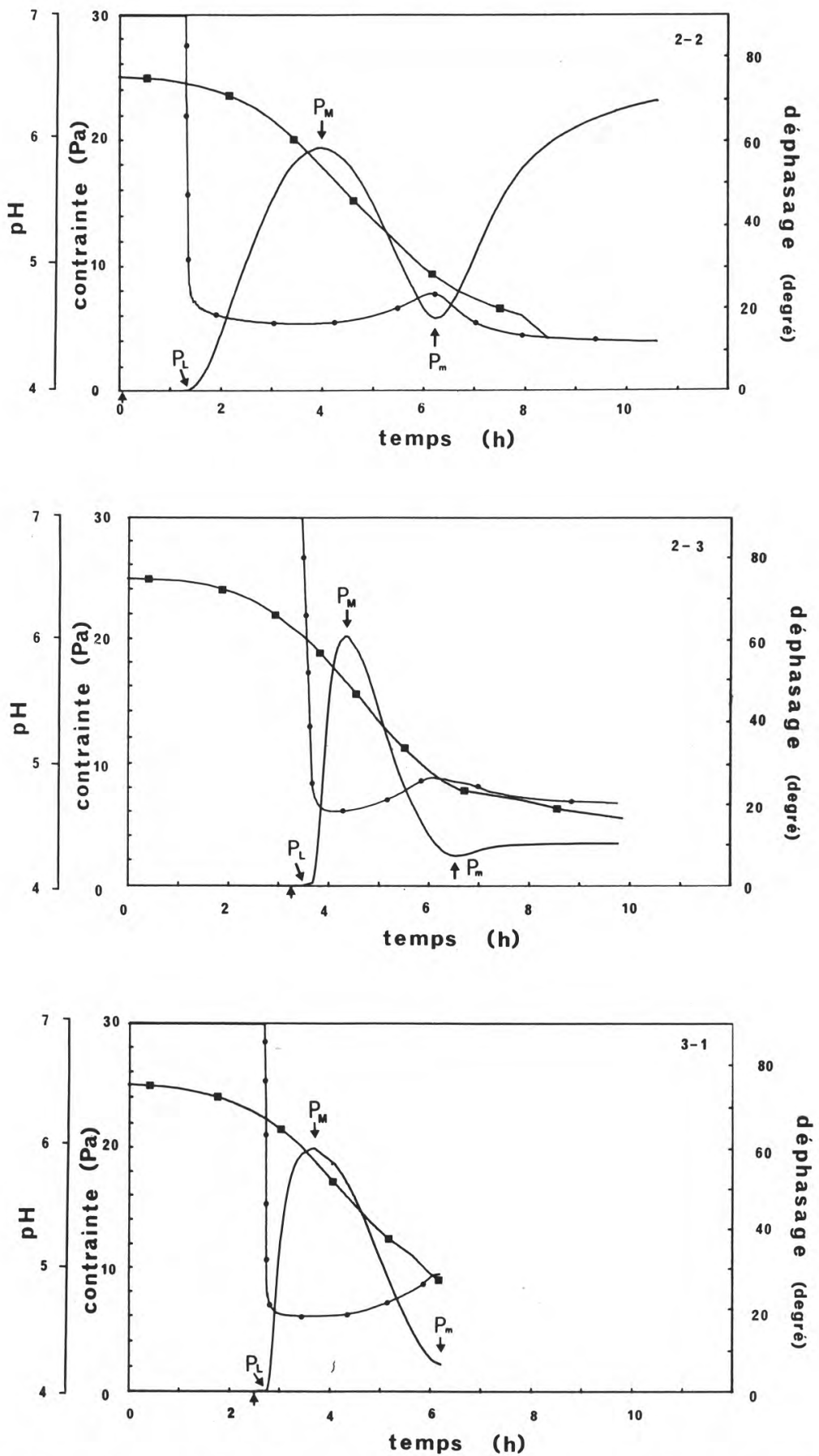

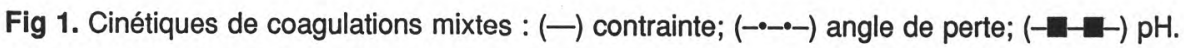
traitement. Les conditions expérimentales correspondantes sont spécifiées dans le tableau I. Combined enzymatic and lactic milk clotting kinetics; (-) stress; (-•--) lag phase; (- - - $)$ pH. ponding experimental conditions are indicated in table $I$. 
Étude multifactorielle en coagulation mixte
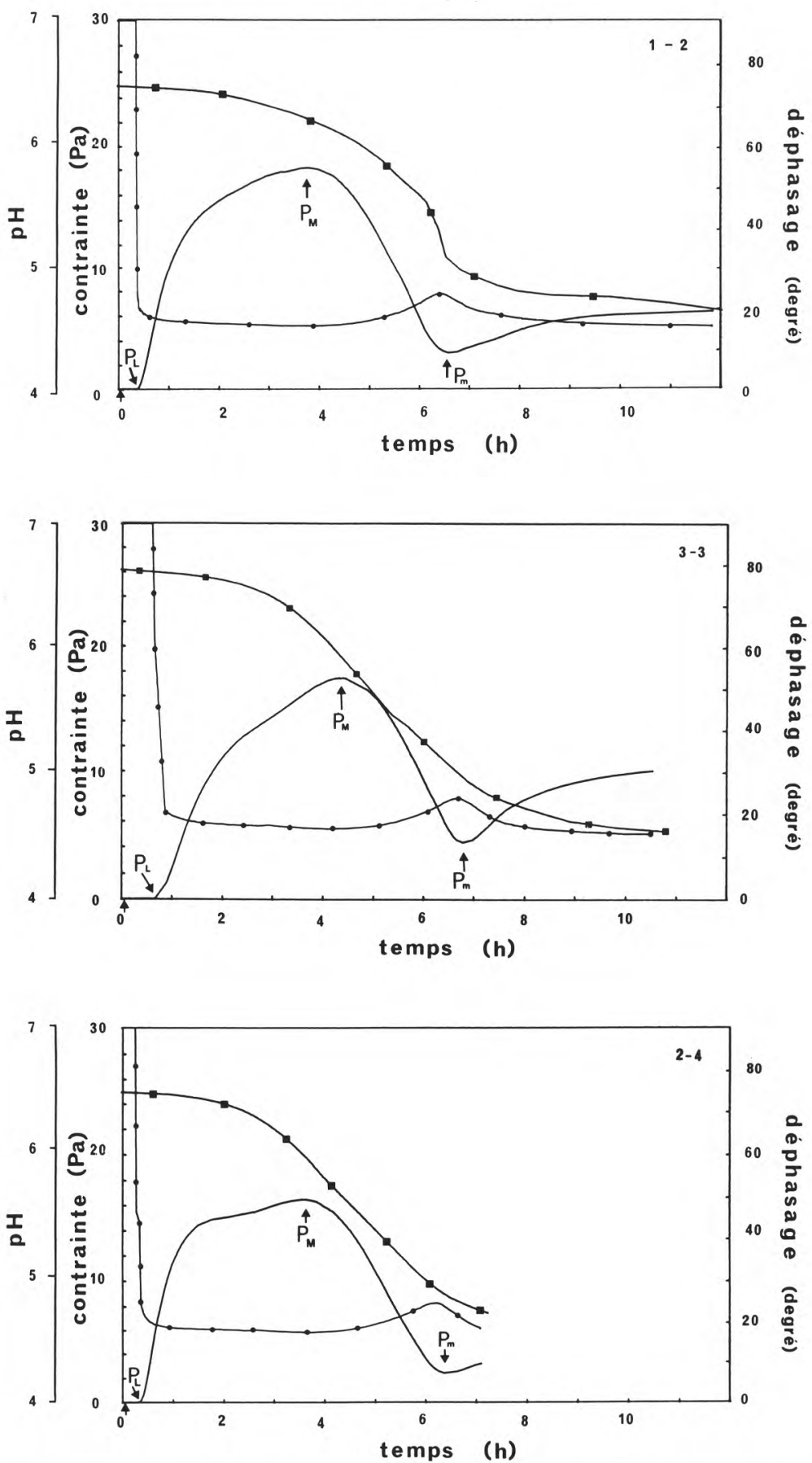

Le temps $t_{0}$ d'addition de la présure est matérialisé par $\boldsymbol{\Delta}$. Chaque graphe est identifié par le code du 
Ce sont les points particuliers (sauf peut-être $\left.P_{6}\right)$ qui résument l'ensemble de l'information contenue dans les courbes, et par suite suffisent à paramétrer celles-ci.

Les grandeurs caractéristiques, dont les variations sont observées dans le cadre du plan d'expérience, sont, d'une part, les coordonnées de ces points, à savoir la contrainte et le temps, d'autre part le $\mathrm{pH}$ et l'angle de perte correspondant. Enfin, la vitesse maximale de raffermissement $V_{\max }$ est définie ici comme la valeur maximale de la dérivée de la contrainte, c'est-à-dire la pente au point d'inflexion de la courbe de contrainte.

II convient de souligner que l'analyse quantitative des résultats a dû être limitée, en pratique, aux caractéristiques des points $P_{L}$ et $P_{M}$ et à $V_{\text {max }}$, compte tenu des perturbations dues à la synérèse qui affectent les mesures après un certain temps, et dans certaines conditions notamment de température, temps qui correspond sensiblement au moment où le point $P_{m}$ est observable.

\section{Le plan d'expérience}

\section{Structure du plan d'expérience}

Le plan d'expérience est un plan factoriel composite centré à 3 facteurs (Box et Wilson, 1951). Les 3 facteurs, température, $\mathrm{pH}$ à l'emprésurage et dose de présure, sont notés respectivement $T$, pHe et Dose. Un essai ou traitement est défini par un niveau donné pour chacun des 3 facteurs.

On a choisi de coder les niveaux des facteurs selon les indications du tableau la. Si les codages des facteurs sont reportés sur 3 axes orthogonaux, chaque essai est représenté par un point. Le plan d'expérience e.t défini par l'ensemble des points suivants, visualisés sur la figure 2 :

- un traitement central $(0,0,0)$ répété 6 fois pour évaluer la reproductibilité des essais,

- les 8 points de coordonnées $( \pm 1, \pm 1, \pm 1)$ qui sont les sommets d'un cube, permettent avec le point central d'évaluer les effets linéaires et les interactions,

- sur chacun des 3 axes, 2 points répartis symétriquement autour de l'origine à une distance de 1,6 . Ces points permettent d'évaluer les effets quadratiques. La valeur de 1,6 permet de prédire la réponse pour un traitement donné avec une variance qui ne dépend que de la distance au point central, propriété dite "d'isovariance par rotation" (Box et Hunter, 1957; Myers, 1971). Cela fait au total 20 traitements, répartis en 3 blocs pour détecter une dérive temporelle éventuelle, et ceci indépendamment des effets des facteurs (tableau lb). $\mathrm{Ce}$ plan d'expérience est une fraction judicieusement choisie du plan complet de $5 \times 5 \times 5$ points ( 3 facteurs à 5 niveaux). II permet d'évaluer les effets des facteurs avec un nombre limité d'expérimentations. Chaque expérimentation est longue (10-12 h) de sorte qu'il est difficile, en pratique, de multiplier le nombre d'essais. En particulier, pour estimer la variabilité en chaque point, il serait nécessaire de faire plusieurs répétitions en chacun d'eux, ce qui augmenterait le nombre d'essais à un niveau qui n'est plus compatible avec les contingences matérielles. Le plan d'expérience est une technique qui autorise un compromis entre l'étude de plusieurs facteurs et l'estimation de la variabilité, ici au point central, l'hypothèse selon laquelle cette variabilité est la même en tous les points du plan pouvant être discutée.

\section{Le modèle général}

On fait l'hypothèse que, sur le plan complet, une grandeur caractéristique $Y$ de la cinétique de coagulation peut être modélisée comme suit :

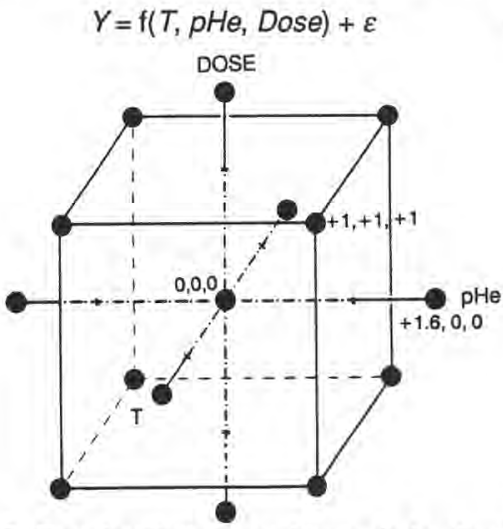

Fig 2. Représentation graphique de la structure du plan d'expérience.

Schematic diagram of the experimental design. 
Tableau I. Le plan d'expérience : la. Correspondance entre niveaux codés et niveaux explicites des facteurs (le 1er chiffre du numéro du traitement désigne le bloc). Ib. Liste des traitements du plan d'expérience. $T=$ température, $\mathrm{pHe}=\mathrm{pH}$ à l'emprésurage, $\mathrm{Dose}=$ dose de présure.

Experimental design: la. Correspondence between coded and explicit levels of experimental factors (the 1st figure of the treatment number designates the block number). Ib. List of experimental plan treatments. $\mathrm{T}=$ temperature, $\mathrm{pHe}=$ renneting $\mathrm{pH}$, Dose = rennet quantity.

la

\begin{tabular}{lccccc}
\hline \multicolumn{1}{c}{ Facteur } & \multicolumn{5}{c}{ Niveau codé } \\
& $-1,6$ & -1 & 0 & +1 & $+1,6$ \\
\hline & 31,4 & 32 & 33 & 34 & 34,6 \\
$\begin{array}{l}\text { Température }\left({ }^{\circ} \mathrm{C}\right) \\
\mathrm{pH} \text { d'emprésurage }\end{array}$ & 5,98 & 6,10 & 6,30 & 6,50 & 6,62 \\
$\begin{array}{l}\text { Dose de présure } \\
\text { (mg/kg lait) }\end{array}$ & 1 & 10 & 25 & 40 & 49 \\
( $\mu$ g chymosine/kg lait) & 4,4 & 44,4 & 111 & 177,5 & 217,5 \\
\hline
\end{tabular}

Ib

Bloc

Traitement

$\begin{array}{llll}n^{\circ} & \mathrm{T}\left({ }^{\circ} \mathrm{C}\right) & \mathrm{pHe} & \text { Dose }(\mathrm{mg} / \mathrm{kg} \text { lait })\end{array}$

Bloc 1

$1-1$

$1-2$

$1-3$

$1-4$

$1-5$

$1-6$

32

32

6,10

6,50

10

34

6,10

40

34

6,50

40

33

6,30

10

33

6,30

25

Bloc 2

2-1

32

6,10

40

2-2

2-3

32

34

6,50

10

2-4

34

2-5

33

2-6

33

6,10

10

6,50

40

$6,30 \quad 25$

Bloc 3

$\begin{array}{ll}3-1 & 34,6 \\ 3-2 & 31,4 \\ 3-3 & 33 \\ 3-4 & 33 \\ 3-5 & 33 \\ 3-6 & 33 \\ 3-7 & 33 \\ 3-8 & 33\end{array}$

6,30

25

6,30

25

6,30

25

$6,62 \quad 25$

$5,98 \quad 25$

$6,30 \quad 49$

$6,30 \quad 1$

$6,30 \quad 25$

$\begin{array}{lll}33 & 6,30 & 25 \\ 33 & 6,30 & 25\end{array}$


où $Y$, qui est aussi appelé réponse, diffère de ( $T, p H e, D o s e)$ appelé réponse théorique (Phan Tan Luu et al, 1983) à cause de l'erreur $\varepsilon$.

À partir de la réponse théorique, on introduit des quantités appelées effets factoriels, qui traduisent les influences respectives sur la réponse expérimentale, des différents facteurs et de leurs interactions.

Plus précisément, on définit ces effets à partir des valeurs de la fonction $f$ sur la grille de $5 \times 5 \times 5$ points correspondant aux niveaux $(-1,6)$ $(-1)(0)(+1)(+1,6)$ de chaque facteur. Ainsi, l'effet factoriel moyen désigne la moyenne de cet ensemble de valeurs de valeurs que l'on notera moy.

Pour un facteur donné, par exemple la température, on peut associer à chacun de ses niveaux la moyenne des valeurs de $f$ correspondantes que l'on notera $f(T, \cdot, \cdot)$ :

$$
f(T, \cdot, \cdot)=\Sigma_{p H e, \text { Dose }} f(T, p H e, \text { Dose }) / 25
$$

La pente de la droite qui représente au mieux la relation entre ces moyennes et la température (fig 3a) est appelée effet factoriel linéaire de la température, et noté lin $T$ (John, 1971). La courbure de la portion de parabole qui ajuste au mieux cette même relation (fig 3a) est appelée effet factoriel quadratique, et noté quad T (John, 1971).

On définit de même les effets linéaire et quadratique des autres facteurs.

Considérons 2 facteurs, Tet $p H$. On associera à chaque couple de niveaux la moyenne notée $f(T, p H e,$.$) . La pente de la droite qui repré-$ sente au mieux l'évolution de cette moyenne en fonction de la température peut dépendre du niveau de $\mathrm{pH}$. Si on représente au mieux cette dépendance entre pente et $\mathrm{pH}$ par une droite, la pente de cette dernière (fig $3 b$ ), notée lin $T$. lin $\mathrm{pH}$ (John, 1971), sera appelée intéraction simple.

Ces différents effets factoriels sont théoriques au même titre que la fonction de réponse $f$ dont ils découlent. On pourrait les estimer si on disposait des $5 \times 5 \times 5$ points du plan complet. Or, en pratique, on ne réalise les expérimentations que sur 20 points judicieusement choisis. Pour pouvoir estimer $f$ sur les autres points de la grille, on fait l'hypothèse que $f$ s'écrit comme un polynôme de degré 2 :

$$
\begin{gathered}
f(T, p H e, \text { Dose })=a_{0}+b_{1} \cdot T+b_{2} \cdot T^{2}+c_{1} \cdot p H e \\
+c_{2} \cdot p H e^{2}+d_{1} \cdot \text { Dose }+d_{2} \cdot \text { Dose }^{2}+\text { e.T.pHe } \\
+f . T \cdot \text { Dose }+g \cdot p H e \cdot \text { Dose }+\varepsilon
\end{gathered}
$$
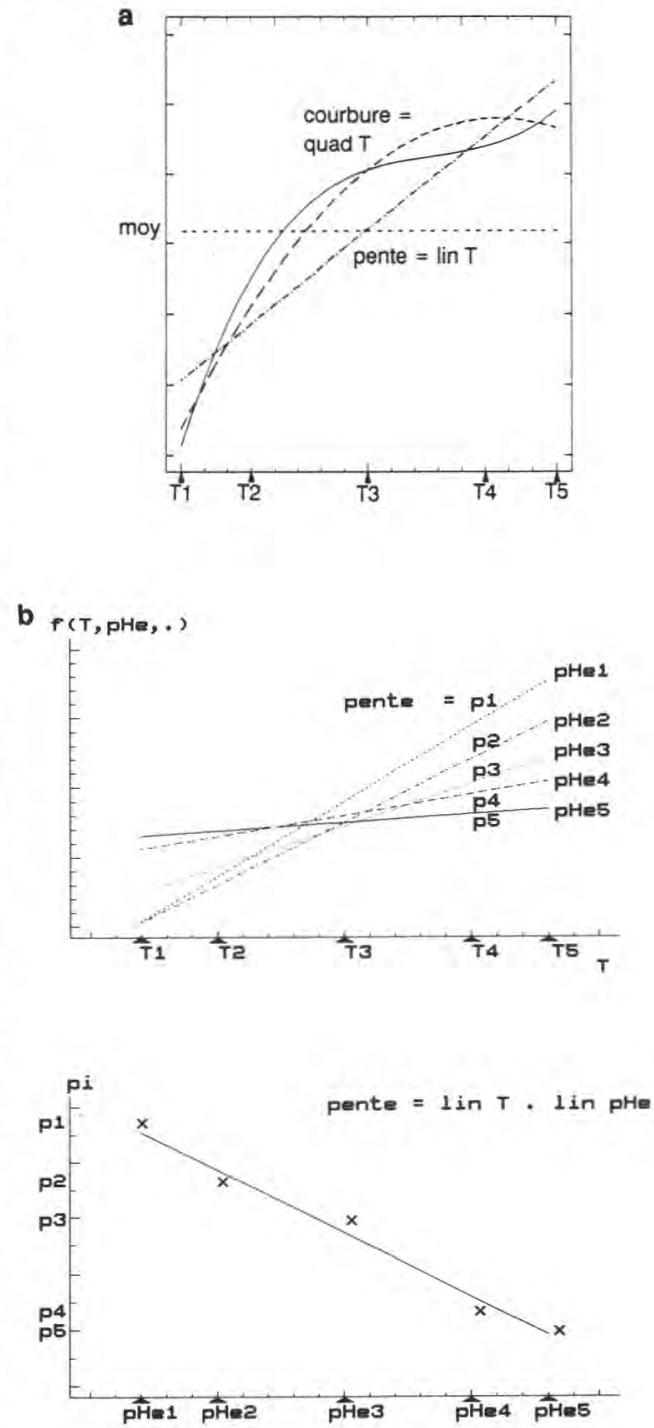

Fig 3. Représentation graphique des effets factoriels (la ligne continue représente la fonction théorique $f(T, \cdot, \cdot): a$, effets moyen $(---)$, linéaire (--.- polynôme de degré 1) et quadratique (- - polynôme de degré 2$)$, b, interaction simple.

Graphic representation of factorial effects (the continued line represents the theoretical fonc-

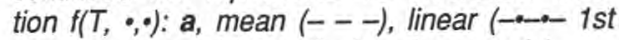
degree polynomial function) and squared (- -2 nd degree polynomial function) effects, $\boldsymbol{b}$, simple interaction. 
Les effets factoriels ne sont pas les coefficients des monômes de cette fonction mais ceux que l'on obtient en réécrivant $f$ en fonction de ce que l'on appelle les polynômes orthogonaux dont les expressions (Kobilinsky, 1988; Durier et Kobilinsky, 1989), pour ce plan particulier, sont données en annexe. Pour rendre les effets factoriels comparables, on doit normer ces polynômes orthogonaux en les divisant par leurs écarts types sur le domaine de variation étudié (cf annexe).

Le modèle réécrit en fonction de ces polynômes orthonormés est :

$$
\begin{aligned}
& Y_{i}=a_{0}^{\prime}+b_{1}^{\prime} \cdot(T / 1,193)+b_{2}^{\prime} \cdot\left(T^{2}-1,424\right) / 0,997 \\
& +c_{1}^{\prime} \cdot(p H e / 1,193)+c_{2}^{\prime} \cdot\left(p H e^{2}-1,424\right) / 0,997 \\
& +d_{1}^{\prime} \cdot(D o s e / 1,193)+d_{2}^{\prime} \cdot\left(D o s e^{2}-1,424\right) / 0,997 \\
& +e^{\prime} \cdot(T / 1,193)(p H e / 1,193) \\
& +f^{\prime} \cdot(T / 1,193)(D o s e / 1,193) \\
& +g^{\prime} \cdot(p H e / 1,193)(\text { Dose } / 1,193)+\varepsilon^{\prime}
\end{aligned}
$$

Les coefficients de ces polynômes sont les effets factoriels recherchés, normés. Le coefficient $a_{0}^{\prime}$ est l'effet factoriel moyen et on remarque que la quantité $a_{0}$ de l'équation (3) correspondant à la réponse au point central, ne coïncide avec la réponse moyenne $a_{0}^{\prime}$ que si les effets quadratiques sont nuls : elle ne présente donc guère d'intérêt.

Le coefficient $b_{1}^{\prime}$ est l'effet factoriel linéaire de la température (lin $T$ ) et $b_{2}^{\prime}$ est l'effet factoriel quadratique (quad T). Enfin, $e$ ' est l'interaction simple de la température et du $\mathrm{pH}$ (lin $\mathrm{T}$ - lin $p H)$.

On reconnaîtra aisément dans les autres coefficients des monômes de l'équation (4) les effets factoriels des autres facteurs.

Les effets factoriels peuvent être estimés directement en ajustant le modèle polynomial (4) aux données expérimentales obtenues pour les 20 traitements, ce qui a été fait ici avec le logiciel Apolor développé par Durier et Kobilinsky (1989). Ce modèle ajusté (4) donne aussi une estimation de $f$ en tout point, en particulier en chacun des $5 \times 5 \times 5$ points de la grille du plan complet.

\section{Les sous-modèles}

Le logiciel Apolor donne les intervalles de confiance aux seuils $90 \%, 95 \%$, et $99 \%$ des effets factoriels estimés. L'écart type résiduel constitue une estimation de la variance de l'erreur.
On construit des sous-modèles en sélectionnant les coefficients significativement différents de 0 , c'est-à-dire ceux dont l'intervalle de confiance à un seuil donné ne contient pas 0 . Ceci conduit, par simple annulation des coefficients non significatifs dans le modèle ajusté (4), à un sous-modèle pour chaque grandeur caractéristique et chaque seuil donné. Les sousmodèles sur lesquels s'appuie l'analyse ont été sélectionnés au seuil de confiance de $95 \%$ parce qu'il convenait bien pour toutes les grandeurs caractéristiques (tableau II). Les sousmodèles permettent de visualiser les effets factoriels sous la forme de surfaces de réponse. Cette procédure est illustrée dans l'annexe pour le cas du temps de latence.

\section{RÉSULTATS DU PLAN D'EXPÉRIENCE}

\section{Reproductibilité des essais}

La reproductibilité des essais est appréciée par l'analyse des 6 traitements identiques correspondant au point central du plan d'expérience. Un modèle spécifique est utilisé pour mettre en évidence les variabilités interbloc et intrabloc pour chaque grandeur caractéristique de la cinétique:

$$
Y_{i j}=\mu+\text { effet } d u b l o c_{i}+e_{i j}
$$

ou ij décrit les 6 couples $(1,5),(1,6),(2,5)$, $(2,6),(3,7),(3,8)$ codant les traitements identiques, et $\mu$ désigne la moyenne des 6 valeurs d'une grandeur caractéristique. Les résultats de l'analyse de variance sont présentés dans le tableau III. Tous les paramètres ont des coefficients de variation inférieurs à $6 \%$, sauf la contrainte au point minimum, la contrainte et l'angle de perte au point $P_{6}$, pour lesquels ils atteignent respectivement les valeurs de 15,50 et $12 \%$. L'analyse de variance (test de Fisher) montre qu'il n'existe pas d'effet bloc significatif, sauf sur le paramètre $\phi_{L}$ pour lequel cet effet est significatif à $3,6 \%$. 
Tableau II. Les sous-modèles au seuil de confiance $95 \%$ pour les principaux paramètres descriptifs de la cinétique de coagulation, avec leur défaut d'ajustement.

Models at $90 \%$ confidence level for principal parameters of milk clotting kinetics, with their lack of fit.

Modèle testé

Signification du défaut d'ajustement

$$
\begin{aligned}
& t_{L}=112,9+836,4 \cdot p H-1492 \cdot D+978,4 \cdot D^{2}-556,5 \cdot D \cdot p H \\
& \phi_{L}=21,10+2,68 \cdot p H+3,83 \cdot \mathrm{pH}^{2} \\
& \tau_{M}=21,4-1,73 \cdot p H+1,19 \cdot D-1,57 \cdot D^{2} \\
& \phi_{M}=16,88+0,75 \cdot \theta-0,534 \cdot D+0,585 \cdot D^{2} \\
& t_{M}=4721,8+4566,3 \cdot p H-1094 \cdot D+1992 \cdot \mathrm{pH}^{2}+1251 \cdot D^{2} \\
& V_{\max }=14,583+1,011 \cdot \theta-8,337 \cdot p H+4,669 \cdot D-3,2313 \cdot p H \cdot D \\
& t_{\theta}=9291-5917 \cdot p H-1330 \cdot \mathrm{pH}^{2} \\
& p H_{L}=6,275+0,225 \cdot p H+0,097 \cdot D-0,094 \cdot D^{2} \\
& p H_{M}=5,914+0,112 \cdot p H+0,152 \cdot D-0,097 \cdot D^{2}
\end{aligned}
$$

++
+++
+
+
+
-
+
+
-

avec $\theta=T-33 ; p H=5 \cdot(p H e-6,30) ; D=($ Dose -25$) / 15$

+ significatif (au seuil 0,01 ), ++ très significatif (seuil $<0,01$ ), +++ hautement significatif (seuil $<0,001$ )

- non significatif (au seuil 0,01).

\section{La matrice de corrélation entre les grandeurs caractéristiques}

La matrice de corrélation entre les grandeurs caractéristiques analysées (tableau IV) est donnée à titre indicatif. II n'est pas possible de tester ici la signification des coefficients puisque les corrélations proviennent en grande partie des effets des facteurs. D'un point de vue qualitatif, un coefficient élevé signifiera que les grandeurs caractéristiques auront le même comportement vis-à-vis des facteurs.

\section{Les effets des facteurs sur les grandeurs caractéristiques de la cinétique de coagulation mixte}

Les variations des grandeurs caractéristiques au point de latence $P_{L}$, temps de latence $t_{L}$, angle de perte $\phi_{L}$ et $p H_{L,}$, en fonction des facteurs étudiés sont présentées sur la figure $4(a, b, c)$. Celles des grandeurs caractéristiques au point maximum $P_{M}$, temps $t_{M}$, contrainte $\tau_{M}$, angle de perte $\phi_{M}$ et $p H_{M}$ sont présentées sur la figure 5 (a, b, c, d). Enfin, la figure 6 présente les résultats concernant la vitesse maximale de raffermissement $V_{\max }$.

On a estimé le défaut d'ajustement d'un modèle (tableau II) par la somme des carrés des écarts entre les moyennes en chaque point répété et les valeurs prédites (Khuri et Cornell, 1987). Ici, il y a un seul point répété, le point central, donc cette somme se réduit à un seul terme : le carré de la différence entre la moyenne et la valeur prédite au point central. L'erreur totale est la somme du défaut d'ajustement et de l'erreur pure, celle-ci étant la somme des carrés des écarts des observations, ici au point central, par rapport à la moyenne de ces observations. La signification du défaut d'ajustement est testée sur le rapport entre celui-ci et l'erreur pure. 
Tableau III. Reproductibilité des essais : moyenne, écart type, coefficient de variation des grandeurs caractéristiques des cinétiques de coagulation. Résultats de l'analyse de variance sur les expériences répétées ( $F=$ statistique de Fisher).

Reproductibility of experimentations: mean, standard deviation, coefficient of variation for the characteristics of the combined enzymatic and lactic milk clotting kinetics. Results of analysis of variance on repeated experimentations ( $\mathrm{F}=$ Fishers' statistic number).

\begin{tabular}{|c|c|c|c|c|c|}
\hline & Moyenne & Ecart type & CV\% & $F$ & Effet bloc \\
\hline Temps de l'emprésurage (s) & 9658 & 570 & 5,9 & 5,44 & NS \\
\hline $\begin{array}{l}\text { Point } P_{L} \\
\text { Temps de latence } t_{L}(\mathrm{~s}) \\
\text { Angle de perte } \phi_{L}(\mathrm{deg}) \\
p H_{L}\end{array}$ & $\begin{array}{r}976 \\
22,43 \\
6,25\end{array}$ & $\begin{array}{l}45 \\
0,1 \\
0,01\end{array}$ & $\begin{array}{l}4,6 \\
0,4 \\
0,16\end{array}$ & $\begin{array}{r}0,703 \\
12,167 \\
0,500\end{array}$ & $\begin{array}{l}\text { NS } \\
\text { NS }\end{array}$ \\
\hline $\begin{array}{l}\text { Point } P_{M} \\
\text { Temps } t_{M}(\mathrm{~s}) \\
\text { Contrainte } \tau_{M}(\mathrm{~Pa}) \\
\text { Angle de perte } \phi_{M}(\mathrm{deg}) \\
\rho H_{M}\end{array}$ & $\begin{array}{c}4519 \\
22,1 \\
17 \\
5,92\end{array}$ & $\begin{array}{c}167 \\
0,6 \\
0,17 \\
0,03\end{array}$ & $\begin{array}{l}3,7 \\
2,7 \\
1 \\
0,56\end{array}$ & $\begin{array}{l}3,135 \\
0,032 \\
1,167 \\
0,924\end{array}$ & $\begin{array}{l}\text { NS } \\
\text { NS } \\
\text { NS } \\
\text { NS }\end{array}$ \\
\hline $\begin{array}{l}\text { Point } P_{m} \\
\text { Temps } t_{m}(\mathrm{~s}) \\
\text { Contrainte } \tau_{m}(\mathrm{~Pa}) \\
\text { Angle de perte } \phi_{m}(\mathrm{deg}) \\
\mathrm{pH} H_{m}\end{array}$ & $\begin{array}{c}13500 \\
4,4 \\
23 \\
4,96\end{array}$ & $\begin{array}{l}314 \\
0,7 \\
0,34 \\
0,05\end{array}$ & $\begin{array}{r}2,3 \\
16,3 \\
1,5 \\
0,9\end{array}$ & $\begin{array}{l}1,684 \\
0,156 \\
0,941 \\
0,054\end{array}$ & $\begin{array}{l}\text { NS } \\
\text { NS } \\
\text { NS } \\
\text { NS }\end{array}$ \\
\hline $\begin{array}{l}\text { Point } P_{6} \\
\text { Contrainte } \tau_{6}(\mathrm{~Pa}) \\
\text { Angle de perte } \phi_{6}(\mathrm{deg}) \\
\mathrm{pH}_{6}\end{array}$ & $\begin{array}{c}9,16 \\
15,5 \\
4,62\end{array}$ & $\begin{array}{l}4,35 \\
1,95 \\
0,04\end{array}$ & $\begin{array}{r}47,5 \\
12,5 \\
0,8\end{array}$ & $\begin{array}{l}0,335 \\
0,319 \\
0,483\end{array}$ & $\begin{array}{l}\text { NS } \\
\text { NS } \\
\text { NS }\end{array}$ \\
\hline $\begin{array}{l}\text { Vitesse maximale de } \\
\text { raffermissement } V_{\max }(\mathrm{Pa} / \mathrm{s})\end{array}$ & 16,18 & 0,48 & 3 & 1,755 & NS \\
\hline
\end{tabular}

* Effet significatif à $3,6 \%$.

Le sous-modèle de $V_{\max }$ présente un défaut d'ajustement négligeable, et tous les résidus (tableau $\mathrm{V}$ ) sont faibles : le modèle est très bon. Le sous-modèle polynomial de $t_{L}$ a un défaut d'ajustement très significatif et 2 résidus très importants, et des valeurs prédites négatives : le modèle n'est pas bon. Le modèle classique décrit plus loin adapté au plan d'expérience (7) et construit sur 19 observations a un défaut d'ajustement négligeable et des résidus faibles dans l'ensemble (tableau V) : le modèle est relativement bon. Le sous-modèle polynomial qui décrit $\phi_{L}$ a un défaut d'ajustement important et 2 résidus importants : le modèle n'est pas très bon. On constate 
Tableau IV. Coefficients de corrélation des grandeurs caractéristiques analysées.

Correlation coefficients between the characteristic parameters of the combined enzymatic and lactic milk coagulation.

\begin{tabular}{lrrrrrrrrr}
\hline & $t_{L}$ & $\phi_{L}$ & $\tau_{M}$ & $\phi_{M}$ & $t_{M}$ & $V_{\max }$ & $t_{e}$ & $p H_{L}$ & $p H_{M}$ \\
\hline$t_{L}$ & 1,00 & & & & & & & & \\
$\phi_{L}$ & 0,09 & 1,00 & & & & & & & \\
$\tau_{M}$ & $-0,78$ & $-0,26$ & 1,00 & & & & & & \\
$\phi_{M}$ & 0,49 & $-0,04$ & $-0,58$ & 1,00 & & & & & \\
$t_{M}$ & 0,65 & 0,40 & $-0,76$ & 0,00 & 1,00 & & & & \\
$V_{\text {max }}$ & $-0,67$ & $-0,25$ & 0,72 & $-0,04$ & $-0,87$ & 1,00 & & \\
$t_{\ominus}$ & $-0,36$ & $-0,31$ & 0,51 & 0,23 & $-0,90$ & 0,82 & 1,00 & & \\
$\mathrm{pH}_{\mathrm{L}}$ & $-0,13$ & 0,33 & $-0,03$ & $-0,58$ & 0,57 & $-0,48$ & $-0,83$ & 1,00 & \\
$\mathrm{pH}_{M}$ & $-0,55$ & 0,07 & 0,29 & $-0,68$ & 0,18 & $-0,10$ & $-0,52$ & 0,85 & 1,00 \\
& & & & & & & & & \\
\hline
\end{tabular}

donc que la qualité d'un modèle n'est pas la même pour toutes les grandeurs caractéristiques.

Le graphique de la figure 7 représente les données du plan d'expérience sous la forme $\left(t_{V}\right)=\log (1 /$ Dose $)$ pour permettre l'examen du modèle classique de HolterFoltmann (Holter, 1932; Foltmann, 1959) entre le temps de latence et la dose de présure, modèle proposé en coagulation enzymatique. En pratique, on a cherché à ajuster les données du plan d'expérience selon le modèle :

$$
t_{L}=K \cdot(1 / D o s e)^{a}
$$

ou

$$
\log \left(t_{L}\right)=\log K+a \cdot \log (1 / \text { Dose })
$$

Si on utilise toutes les données (20 observations), on trouve $a=0,72$ (écart type = $0,05)$. Le paramètre $K$ du modèle dépend des monômes $p H$ et $p H^{R}$, et très faiblement des monômes $T$ et $T 2$. Le défaut d'ajustement du modèle, estimé par la différence entre la moyenne des observations au point central (seul point répété) et la valeur donnée par le modèle (Khuri et Cornell, 1987) est moyen; l'une des observations (traitement $3-6$ ) a un résidu très important $(-3,22)$, qui montre qu'elle ne peut pas être ajustée par ce modèle.

Si on supprime cette donnée, qui correspond à la plus faible dose et au temps de latence le plus long, on trouve $a=0,96$ (écart type $=0,06$ ) qui peut donc être considéré comme n'étant pas significativement différent de 1. La dépendance négligeable du coefficient $K$ par rapport au facteur $T$, comparée à sa dépendance vis-à-vis du $\mathrm{pH}$, conduit à éliminer les termes en $T$ et $T^{2}$ du modèle qui s'écrit :

$$
\begin{gathered}
\log t_{L}=\log K\left(p H, p H^{R}\right) \\
+0,96 \log (1 / \text { Dose })
\end{gathered}
$$

Le défaut d'ajustement de ce modèle est négligeable, ce qui se traduit par des résidus faibles (tableau $\mathrm{V}$ ). On pose alors $a=1$ et on réajuste les données, ce qui donne:

$\log K=10,15+0,49 \cdot p H+0,11 \cdot p H^{R}$

ou

$K(p H)$

$=\exp \left(10,15+0,49 \cdot p H+0,11 \cdot p H^{R}\right)(10)$

les $\mathrm{pH}$ étant considérés en valeurs codées. 
Tableau V. Tableau des valeurs observées et des résidus réduits $(\varepsilon)$ (divisés par leur écart type) des grandeurs caractéristiques; résidus réduits de log $\left(t_{L}\right)$ pour le modèle de Holter-Foltmann $\left(\varepsilon^{\prime}\right)$.

Table of observed values and residual values $\varepsilon$ (divided by their standard error) for the characteristic parameters; residual values of $\log \left(\mathrm{t}_{\mathrm{L}}\right)$ for the Holter-Foltmann's model $\left(\varepsilon^{\prime}\right)$.

\begin{tabular}{|c|c|c|c|c|c|c|c|c|c|c|}
\hline$n^{\circ}$ & $t_{L}$ & $\varepsilon$ & & $\varepsilon^{\prime}$ & $\phi_{\mathrm{L}}$ & $\varepsilon$ & $\tau_{M}$ & $\varepsilon$ & $\phi_{M}$ & $\varepsilon$ \\
\hline $1-1$ & 1737 & $-0,87$ & \multicolumn{2}{|c|}{$-0,15$} & 21,6 & 0,16 & 21,8 & 0,82 & 17,0 & $-0,45$ \\
\hline $1-2$ & 1419 & 1,66 & \multicolumn{2}{|c|}{1,59} & 19,9 & $-1,76$ & 18,2 & $-0,94$ & 16,1 & $-0,06$ \\
\hline $1-3$ & 452 & 0,62 & \multirow{2}{*}{\multicolumn{2}{|c|}{$\begin{array}{r}0,18 \\
-0,55\end{array}$}} & 24,5 & 0,95 & 22,2 & $-0,57$ & 18,1 & 1,08 \\
\hline $1-4$ & 4395 & $-1,20$ & & & 23,3 & $-0,84$ & 17,4 & 0,16 & 17,9 & $-1,82$ \\
\hline $1-5$ & 977 & $-0,05$ & \multicolumn{2}{|c|}{$-0,47$} & 22,5 & 0,70 & 21,9 & 0,15 & 17,1 & 0,57 \\
\hline $1-6$ & 942 & $-0,13$ & \multicolumn{2}{|c|}{$-0,77$} & 22,7 & 0,75 & 22,2 & 0,34 & 17,1 & 0,57 \\
\hline $2-1$ & 425 & 0,37 & \multicolumn{2}{|c|}{$-0,33$} & 22,9 & 0,40 & 22,8 & $-0,08$ & 16,4 & 0,74 \\
\hline $2-2$ & 5178 & 0,61 & \multicolumn{2}{|c|}{0,82} & 21,7 & $-1,39$ & 19,4 & 1,63 & 16,5 & $-1,48$ \\
\hline $2-3$ & 1573 & $-1,48$ & \multicolumn{2}{|c|}{$-0,99$} & 23,6 & 0,59 & 20,2 & $-0,23$ & 18,3 & $-0,79$ \\
\hline $2-4$ & 1269 & 1,09 & \multicolumn{2}{|c|}{0,66} & 23,8 & $-0,82$ & 16,5 & $-2,06$ & 17,7 & 0,28 \\
\hline $2-5$ & 911 & $-0,35$ & \multicolumn{2}{|c|}{$-1,04$} & 22,6 & 0,61 & 21,7 & 0,09 & 17,2 & 0,89 \\
\hline $2-6$ & 1011 & $-0,14$ & \multicolumn{2}{|c|}{$-0,19$} & 22,5 & 0,58 & 22,5 & 0,60 & 16,9 & 0,26 \\
\hline $3-1$ & 1016 & $-0,51$ & \multicolumn{2}{|c|}{$-0,15$} & 23,4 & 0,06 & 19,9 & $-0,67$ & 18,3 & 0,14 \\
\hline 3-2 & 1453 & 0,39 & & 78 & 21,0 & $-0,55$ & 21,3 & 0,21 & 15,9 & 0,14 \\
\hline $3-3$ & 2512 & $-0,21$ & & & 47,1 & 3,74 & 17,5 & $-0,50$ & 16,6 & $-0,90$ \\
\hline 3-4 & 700 & 1,82 & & 30 & 22,7 & $-2,27$ & 22,0 & $-1,24$ & 17,3 & 0,54 \\
\hline $3-5$ & 500 & $-2,68$ & & 44 & 23,3 & 0,04 & 21,9 & 2,82 & 17,0 & $-2,07$ \\
\hline $3-6$ & 6911 & 2,31 & & & 23,0 & $-0,04$ & 12,7 & $-2,16$ & 20,5 & 3,36 \\
\hline 3-7 & 1022 & $-0,49$ & & 10 & 22,1 & $-0,27$ & 22,8 & 1,14 & 16,7 & $-0,69$ \\
\hline $3-8$ & 991 & $-0,56$ & & 35 & 22,2 & $-0,24$ & 21,6 & 0,39 & 17,0 & $-0,08$ \\
\hline$n^{\circ}$ & $t_{M}$ & $\varepsilon$ & $\mathrm{V}_{\max }$ & $\varepsilon$ & $t_{\theta}$ & $\varepsilon$ & $\mathrm{pH}_{\mathrm{L}}$ & $\varepsilon$ & $\mathrm{pH}_{\mathrm{M}}$ & $\varepsilon$ \\
\hline $1-1$ & 4557 & $-0,36$ & 14,9 & 0,59 & 12440 & $-0,59$ & 5,96 & 1,07 & 5,66 & 0,72 \\
\hline $1-2$ & 13453 & 2,02 & 5,6 & $-1,14$ & 160 & $-0,89$ & 6,49 & $-0,56$ & 6,24 & 1,51 \\
\hline $1-3$ & 2589 & $-0,09$ & 32,4 & 0,34 & 14525 & 0,80 & 6,03 & $-0,69$ & 5,80 & $-1,76$ \\
\hline $1-4$ & 13331 & $-0,79$ & 4,3 & $-1,49$ & 170 & $-0,88$ & 6,44 & 1,48 & 5,87 & 0,52 \\
\hline $1-5$ & 4666 & $-0,47$ & 15,6 & 0,54 & 9635 & 0,58 & 6,25 & $-0,66$ & 5,92 & $-0,73$ \\
\hline $1-6$ & 4848 & $-0,26$ & 15,9 & 0,74 & 10185 & 0,94 & 6,26 & $-0,53$ & 5,96 & $-0,18$ \\
\hline $2-1$ & 2809 & 0,45 & 29,8 & $-0,36$ & 15136 & 1,09 & 6,05 & $-0,41$ & 5,79 & $-1,03$ \\
\hline $2-2$ & 14113 & 0,43 & 3,3 & $-0,79$ & 220 & $-0,97$ & 6,44 & 1,48 & 5,80 & 0,34 \\
\hline $2-3$ & 3804 & $-1,00$ & 16.7 & 0,21 & 11743 & $-1,18$ & 5,95 & 0,93 & 5,66 & 1,61 \\
\hline $2-4$ & 12647 & 1,31 & 6,9 & $-1,96$ & 177 & $-1,00$ & 6,49 & $-0,56$ & 6,02 & $-0,93$ \\
\hline $2-5$ & 4183 & $-0,77$ & 17,2 & 1,47 & 11084 & 1,40 & 6,26 & $-0,53$ & 5,95 & 0,49 \\
\hline $2-6$ & 4544 & $-0,35$ & 16,1 & 0,73 & 9811 & 0,58 & 6,24 & $-0,79$ & 5,88 & $-0,48$ \\
\hline $3-1$ & 4293 & 0,07 & 16,6 & 0,15 & 8990 & $-0,77$ & 6,23 & 0,08 & 5,91 & 0,73 \\
\hline $3-2$ & 5030 & 0,88 & 12,5 & $-0,49$ & 10130 & $-0,06$ & 6,20 & $-0,30$ & 5,84 & $-0,21$ \\
\hline $3-3$ & 15303 & $-2,20$ & 3,2 & 1,30 & 207 & 2,66 & 6,59 & 0,08 & 5,95 & $-1,30$ \\
\hline $3-4$ & 2725 & 1,16 & 25,5 & $-1,93$ & 15533 & $-0,70$ & 5,91 & 0,67 & 5,67 & $-0,10$ \\
\hline $3-5$ & 3975 & $-2,82$ & 22,1 & $-0,11$ & 10327 & 0,07 & 6,26 & 2,25 & 5,95 & 1,97 \\
\hline $3-6$ & 10258 & 1,77 & 6,0 & $-0,96$ & 12519 & 1,45 & 5,63 & $-3,66$ & 5,24 & $-2,48$ \\
\hline $3-7$ & 4469 & 0,26 & 16,3 & 0,99 & 8701 & $-0,96$ & 6,24 & 0,20 & 5,89 & 0,46 \\
\hline $3-8$ & 4406 & 0,19 & 16,0 & 0,80 & 8535 & $-1,06$ & 6,25 & 0,33 & 5,90 & 0,60 \\
\hline
\end{tabular}

$\varepsilon$ : résidus réduits pour les sous-modèles

$\varepsilon^{\prime}$ : résidus réduits de $\log \left(t_{L}\right)$ pour le modèle classique. 

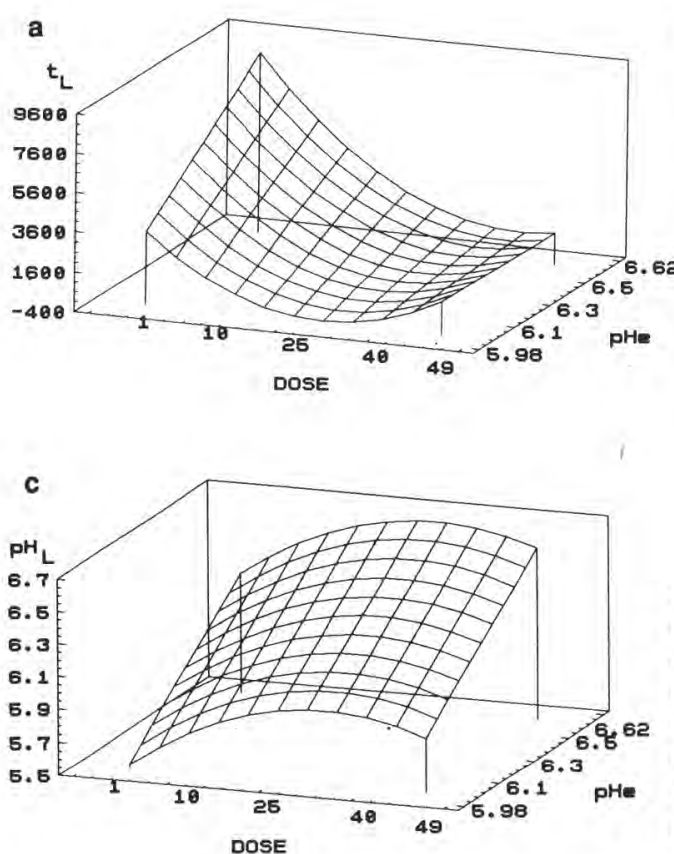

Le modèle reliant le temps de latence et la dose de présure, obtenu à partir de 19 observations, s'écrit finalement :

$$
t_{L}=K(p H) / \text { Dose }
$$

où $\mathrm{K}(\mathrm{pH})$ correspondrait à une constante d'activité de l'enzyme coagulante, fonction du $\mathrm{pH}$.

\section{Évolution des cinétiques pour un temps de latence donné}

Les courbes d'isoréponse du temps de latence (fig $8 \mathrm{a}$ ) ont été établies à partir du modèle (11), celles de la contrainte maximale (fig $8 b$ ) et de la vitesse maximale de raffermissement (fig $8 \mathrm{c}$ ) ont été établies à partir des sous-modèles donnés dans le tableau III. L'examen de ces courbes révèle d'une part qu'un même temps de latence peut être obtenu à partir de diffé-

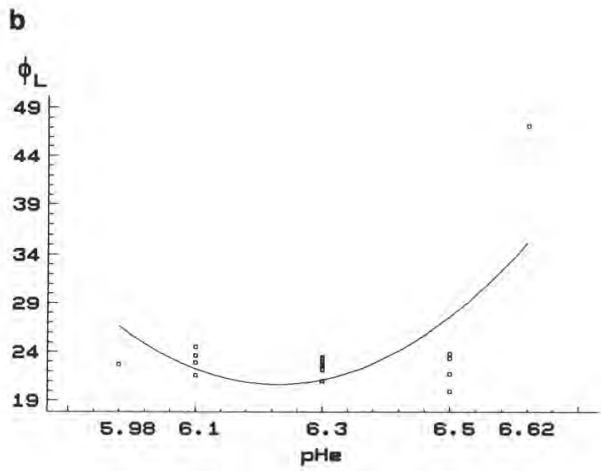

Fig 4. Surfaces de réponse des grandeurs caractéristiques au point de latence $P_{L} . \mathbf{a}: t_{L} ; \mathbf{b}$ : $\phi_{L} ; \mathbf{c}: p H_{L}$. Chaque surface de réponse est la représentation graphique du modèle établi pour chaque grandeur caractéristique, modèle donné dans le tableau III.

Response surface plots of characteristics at lag point $\mathrm{P}_{\mathrm{L}} \cdot \boldsymbol{a}: \mathrm{t}_{\mathrm{L}} ; \boldsymbol{b}: \phi_{\mathrm{L}} ; \boldsymbol{c}: \mathrm{pH}_{\mathrm{L}}$.

rentes combinaisons des 2 facteurs Dose et $\mathrm{pHe}$, d'autre part que les caractéristiques de la phase de raffermissement peuvent être nettement différentes pour un même temps de latence. Ainsi, un $\mathrm{pHe}$ élevé peut être compensé par une Dose forte pour obtenir le même temps de latence (fig 8a); en outre, plus le pHe est élevé, moins une augmentation de la Dose au-delà d'une certaine valeur, fonction du $\mathrm{pHe}$, agit sur la vitesse maximale de raffermissement (fig $8 \mathrm{c}$ ) alors qu'elle agit encore sur le temps de latence (fig 8a).

Pour un même temps de latence, par exemple $t_{L}=1000 \mathrm{~s}$, pour lequel la courbe d'isoréponse a été reportée sur les figures $8 \mathrm{~b}$ et $8 \mathrm{c}$, la contrainte maximale peut être, soit élevée (à $\mathrm{pHe}$ faible et Dose faible), soit faible ( $p H e$ élevé et Dose élevée) tandis que la vitesse maximale de raffermissement est respectivement soit forte, soit faible. 

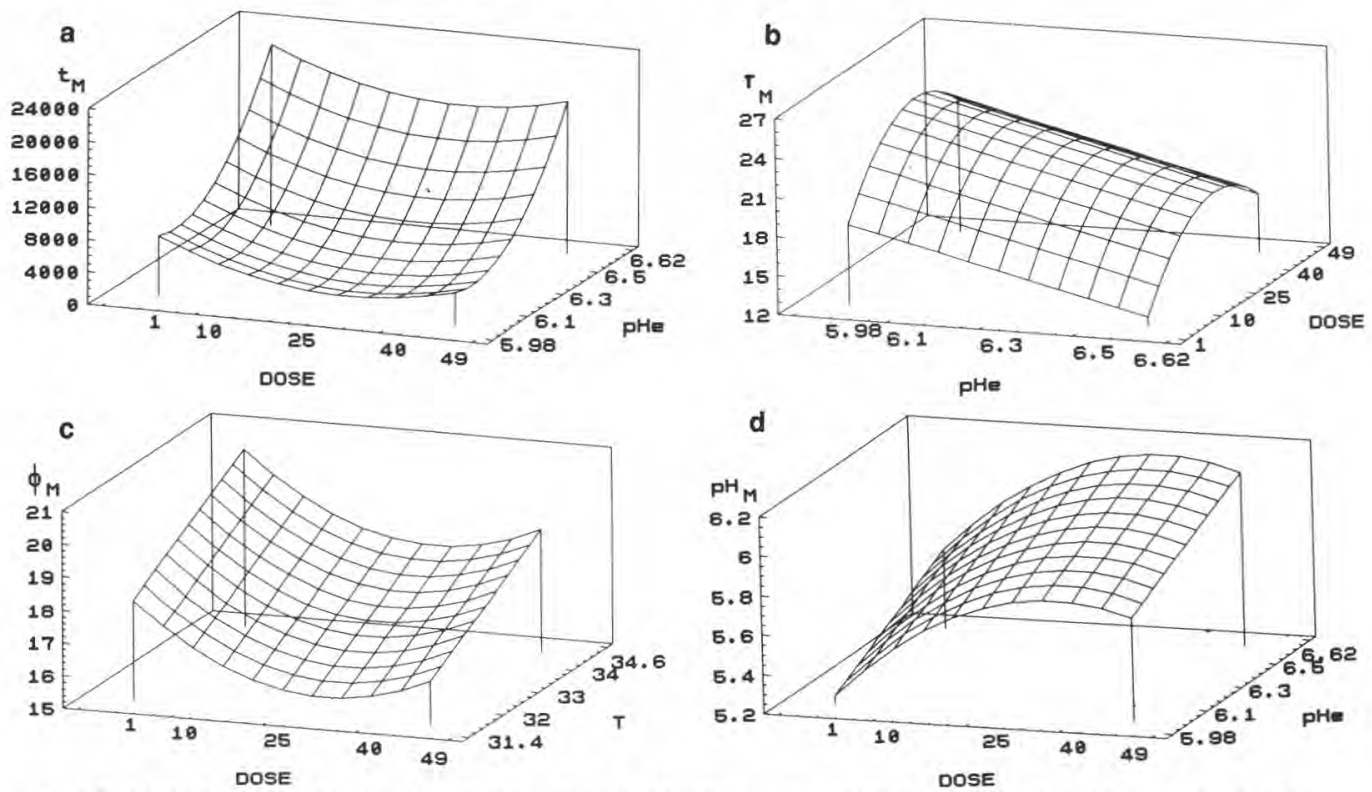

Fig 5. Surfaces de réponse des grandeurs caractéristiques au point maximum. $\mathbf{a}: t_{M} ; \mathbf{b}: \tau_{M} ; \mathbf{c}: \phi_{M} ; \mathbf{d}$ : $\mathrm{pH}_{\mathrm{M}}$.

Response surface plots of characteristics at maximum point $P_{M}: \mathbf{a}: t_{M} ; \mathbf{b}: \tau_{M} ; \mathbf{c}: \phi_{M} ; \mathbf{d}: \mathrm{pH}_{M}$.

\section{DISCUSSION}

Lorsque l'emprésurage et la prise ont lieu à un $\mathrm{pH}$ plutôt élevé, la cinétique de formation du coagulum du fait de l'action enzymatique, se manifeste jusqu'au début de la

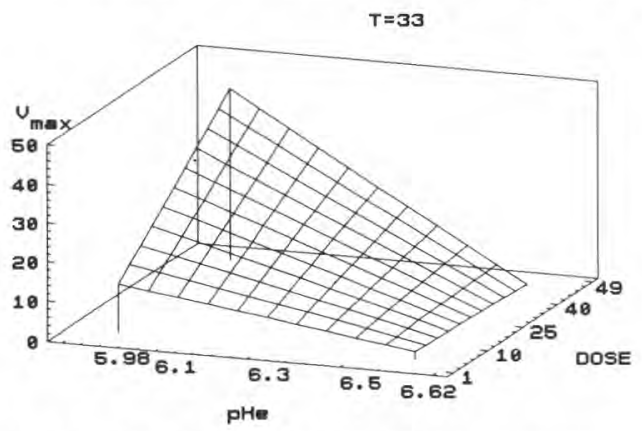

Fig 6. Surface de réponse de la vitesse maximale de raffermissement à $33^{\circ} \mathrm{C}$.

Response surface plots of maximum firming rate at $33^{\circ} \mathrm{C}$. phase plateau, avant que la cinétique de déminéralisation du coagulum consécutive à l'acidification n'induise une diminution de la "fermeté» (fig $1 \mathrm{~d}, \mathrm{e}, \mathrm{f}$ ).

Par ailleurs, suivant les conditions opératoires notamment celles de la température, la quatrième phase de la coagulation mixte ne peut pas être observée (fig 1 b, c, f) : la synérèse provoque la formation d'un film liquide entre le cylindre de mesure et le coagulum, ce qui interdit les mesures.

\section{La reproductibilité des essais}

L'analyse statistique des résultats des 6 traitements identiques destinés à mettre en évidence l'effet des facteurs non contrôlés (opérateur, durée d'incubation de la souche, etc.) montre que la reproductibilité est satisfaisante jusqu'au point minimum 


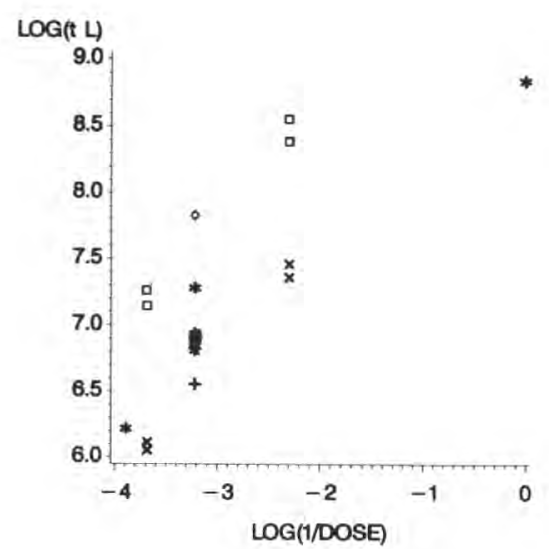

Fig 7. Représentation graphique de $\log t_{\mathrm{L}}$ en fonction de log (1/Dose : $(++) \mathrm{pHe}=5,98(x \times)$ $\mathrm{pHe}=6,1\left(^{\star *}\right) \mathrm{pHe}=6,3(\square \square) \mathrm{pHe}=6,5(\star \star)$ $\mathrm{pHe}=6,62$.

Plot of log $t_{L}$ vs log (1/Dose): $(++) p H e=5,98$ $(x x) \mathrm{pHe}=6,1\left(^{*}\right) \mathrm{pHe}=6,3$ (口) $\mathrm{pHe}=6,5$ (*) $\mathrm{pHe}=6,62$.

$P_{m}$ : les coefficients de variation des temps $t_{L}, t_{M}, t_{m}$, sont inférieurs à $5 \%$; celui de la contrainte $\tau_{M}$ est inférieur à $3 \%$ alors que celui de $\tau_{m}$ est supérieur à $15 \%$; ceux des angles de pente $\phi_{L}, \phi_{M}, \phi_{m}$ sont inférieurs ou égaux à $1,5 \%$; enfin, ceux des $\mathrm{pH}$ sont inférieurs à $0,6 \%$. A partir du point minimal $P_{m}$, on peut observer visuellement un détachement plus ou moins important du coagulum par rapport à la paroi de la cuve de l'appareil de mesure, détachement qui peut être imputé à des phénomènes de synérèse. Par suite, on peut supposer que la valeur de $\tau_{m}$ est le plus souvent sous-estimée, tout en étant caractérisée par une variabilité importante. Les paramètres mesurés après ce point sont aussi variables sinon plus, de sorte que les informations relatives à cette phase de la coagulation mixte ne peuvent raisonnablement ici être exploitées que d'une façon qualitative. Le temps d'emprésurage $t_{0}$ présente un écart type relativement impor- tant $(570 \mathrm{~s} ; C V \%=5,9 \%)$. Ce temps est celui que mettent les ferments lactiques pour acidifier le lait jusqu'au pH d'emprésurage $(6,30)$. Toutefois, les résultats obtenus sur les paramètres de la cinétique ne montrent pas d'incidence sur le déroulement de la coagulation : le pHe évolue parallèlement pour les 6 traitements, l'écart type du $\mathrm{pHe}$ des 4 points caractéristiques est voisin de l'erreur de mesure sur le $\mathrm{pH}$ tandis que les temps associés ont des coefficients de variation compris entre 2 et $5 \%$.

\section{Le plan d'expérience}

Le choix des traitements (niveaux et combinaisons des facteurs) est associé au modèle polynomial de degré 2 retenu pour ajuster l'ensemble des grandeurs caractéristiques des cinétiques de coagulation mixte. Par suite, le modèle classique de Holter (1932) et Foltmann (1959) qui lie le temps de latence et la dose de présure, proposé pour les cinétiques de coagulation enzymatique, n'a pas été privilégié au départ. Toutefois, les données du plan d'expérience ont aussi été examinées avec ce modèle : les résultats sont présentés cidessus et discutés plus loin.

Un résultat notable du plan d'expérience est l'absence d'effet significatif de la température dans la gamme étudiée (32$34^{\circ} \mathrm{C}$ ) sur la plupart des paramètres descriptifs de la coagulation. Seuls la vitesse maximale de raffermissement, l'angle de perte au point maximum et les coordonnées du point minimum présentent un effet "température", qui reste de toute façon limité quand on le compare à l'effet du $\mathrm{pHe}$ et de la Dose (tableau II).

Une diminution du temps de latence est observée par Ramet et Weber (1980) et Lee (1986) entre 20 et $40^{\circ} \mathrm{C}$, par Bohlin et al (1984) entre 31 et $35^{\circ} \mathrm{C}$, par Zoon et al (1988b) entre 30 et $35^{\circ} \mathrm{C}$. Mais entre 40 et 


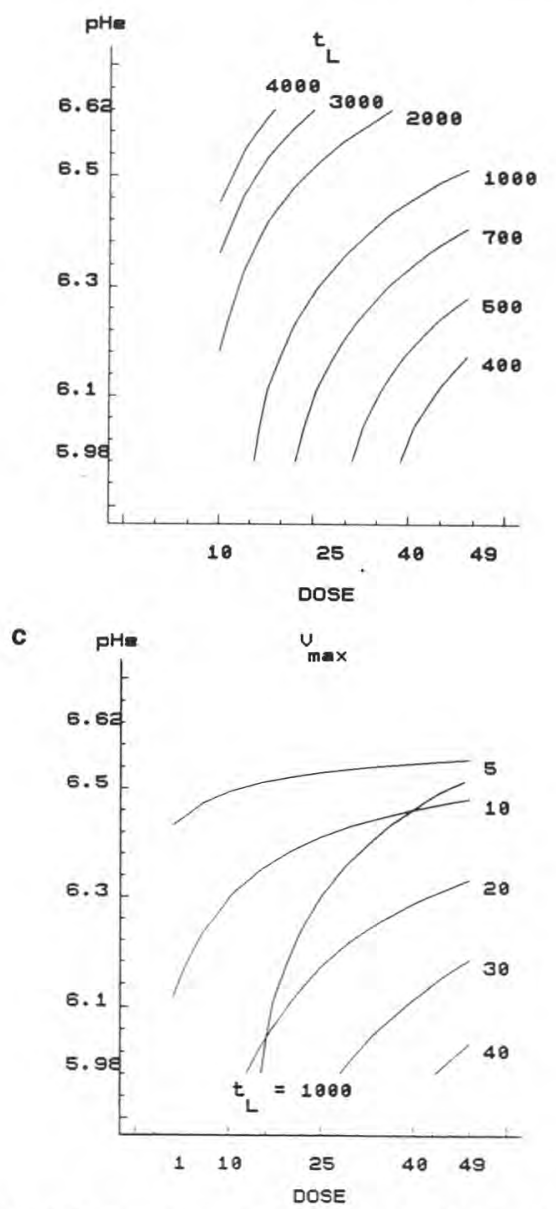

$50{ }^{\circ} \mathrm{C}$, celui-ci augmenterait (Ramet et Weber, 1980). Collin et al (1977) ont mis en évidence qu'une variation de $0,2^{\circ} \mathrm{C}$ autour de $30{ }^{\circ} \mathrm{C}$ induit une variation de $15 \%$ du temps de floculation visible alors que l'examen des autres résultats permet d'estimer un pourcentage de variation, due à la température, du temps de latence cette variation serait de $15 \%$ entre 32 et $34{ }^{\circ} \mathrm{C}$ selon Ramet et Weber (1980), de $25 \%$ entre 31 et $35^{\circ} \mathrm{C}$ selon Bohlin et al (1984), et $10 \%$ entre 30 et $35^{\circ} \mathrm{C}$ selon Zoon et al (1988b). Ces derniers auteurs ne donnent aucune indication sur la reproductibilité des résultats et donc sur leur degré de signification.

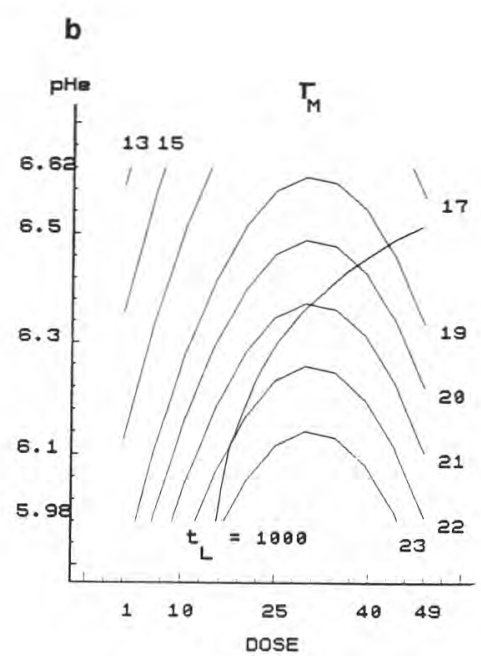

Fig 8. Courbes d'isoréponse a du temps de latence $t_{L}$ avec le modèle classique, $b$ de la contrainte maximum $\tau_{M}$, et $\mathbf{c}$ de la vitesse maximale de raffermissement $V_{\max }$ avec les modèles polynomiaux.

Contour plots of a lag time $t_{L}$ (values calculated from the classical model), $\boldsymbol{b}$ maximum stress $\tau_{M}$, $c$ maximum firming rate $V_{\max }$ (values calculated from the polynomial models).

Les résultats donnés en annexe pour $t_{L}$ correspondent pour cette expérience à une variation de $11 \%$ entre 32 et $34^{\circ} \mathrm{C}$, variation dont l'amplitude est comparable à la majorité des résultats de la littérature. Cet effet n'est pas statistiquement significatif, d'une part en raison d'un certain manque de sensibilité de l'appareil utilisé, ce qui se traduit par un écart type résiduel notable (tableau A-1), d'autre part en raison du petit nombre d'essais ( 5 pour les températures supérieures à $33^{\circ} \mathrm{C}$, et 5 pour les températures inférieures à $33^{\circ} \mathrm{C}$ ).

Bohlin et al (1984) ont observé une augmentation de la vitesse de raffermissement entre 31 et $35^{\circ} \mathrm{C}$, de même que Zoon et al (1988b) entre 25 et $35^{\circ} \mathrm{C}$. 
L'influence de la température observée ici reste tout à fait limitée sur $\mathrm{V}_{\max }$; son sens de variation est en accord avec les résultats de la littérature. La cinétique enzymatique est moins sensible à la température que la cinétique de floculation et de formation du réseau protéique (Walstra et Van Vliet, 1986). II est probable que l'effet de la température qui apparaît ici correspond bien à un effet sur les liaisons, notamment hydrophobes, qui conduirait à un réarrangement spatial intramicellaire avec augmentation de la voluminosité des micelles (Van Vliet et al, 1989); l'effet serait ici limité seulement parce que l'étendue explorée des températures est faible.

Selon les auteurs, le module élastique maximal augmente (Tokita et al, 1982, $1983: 15-35^{\circ} \mathrm{C}$; Bohlin et al, $1984: 31$ et $35{ }^{\circ} \mathrm{C}$ ) ou diminue (Ramet et Weber, 1980: 20-50 ${ }^{\circ} \mathrm{C}$; Lee, $1986: 20-40^{\circ} \mathrm{C}$; Walstra et Van Vliet, $1986: 20-40^{\circ} \mathrm{C}$; Zoon et al, 1988b : $\left.25-35^{\circ} \mathrm{C}\right)$. Cette contradiction a déjà été notée (Zoon et al, 1988b). Quant à la tangente de l'angle de perte, elle augmente avec la température (Walstra et Van Vliet, 1986; Zoon et al, 1988b).

Pour une faible variation de la température, nous n'observons pas d'effet significatif sur $\tau_{M}$ contrairement à $\phi_{M}$. On peut penser qu'ici, l'étroitesse du domaine de température exploré n'induit pas de variation significative sur la contrainte maximale, qui représente en quelque sorte la résultante de la composante élastique ( $G^{\prime}$ ) et de la composante visqueuse ( $\left.G^{\prime \prime}\right)$. Le faible effet sur l'angle de perte $\phi_{M}$ pourrait signifier néanmoins que les effets de la température sur G' d'une part, G" d'autre part, sont suffisamment différents pour se manifester sur le rapport $G^{\prime \prime} / G^{\prime}$ qui n'est autre que $\operatorname{tg} \phi$.

Au point minimum $P_{m}$, il est raisonnable de penser que l'effet de la température est associé au phénomène de synérèse; celleci est accélérée par une augmentation même faible de la température (Walstra et al, 1985; Van Den Bijgaart, 1988), il en résulte une dégradation des conditions de l'essai : le coagulum se décolle en partie des parois de la cuve, ce qui se traduit pour l'échantillon par un cisaillement variable dans l'espace et le temps, et la mesure effectuée perd en partie sa pertinence.

La dose de présure, en coagulation mixte, a un effet linéaire et quadratique sur l'ensemble des paramètres caractéristiques des points $P_{L}$ et $P_{M}$, mais pas d'effet sur l'angle de perte $\phi_{L}$ (tableau II).

II est admis que le temps de latence diminue avec l'augmentation de la dose de présure.

Le sous-modèle polynomial établi à partir du plan d'expérience (tableau II) montre l'existence d'un effet quadratique de la Dose sur le temps de latence. II convient toutefois de noter que l'approximation quadratique conduit à modéliser la réponse d'une grandeur au moyen d'une parabole, ce qui pose un problème particulier : en effet, une parabole "retombe" et ne permet pas d'ajuster des données expérimentales à une asymptote. Cette approximation est à l'origine ici d'un artefact quant à l'effet de la Dose : elle génère, en effet, des temps de latence négatifs. L'effet quadratique de la Dose sur le temps de latence doit donc être interprété ici plutôt comme révélateur de l'existence d'un plateau pour les fortes doses de présure. On a un effet comparable en ce qui concerne la contrainte maximale discutée plus loin. De plus, le plan d'expérience met en évidence une interaction importante de la dose de présure avec le $\mathrm{pH}$ sur le temps de latence (figs $4 \mathrm{a}$ et $8 \mathrm{a}$; tableau II). $\mathrm{A} \mathrm{pH}$ $6,6, t_{L}$ diminue avec l'augmentation de la Dose; à $\mathrm{pH} \mathrm{6,2} \mathrm{et} \mathrm{en-dessous,} t_{L}$ ne dimi- 
nue plus au-delà d'une certaine Dose (fig $8 b)$. L'examen détaillé des résultats de McMahon et al (1984) révèle aussi un effet quadratique de la Dose sur le temps de latence, mais ne permet pas de dégager des interactions avec le $\mathrm{pH}$ qui est maintenu constant.

Le modèle classiquement utilisé pour décrire la relation entre le temps de latence et la dose de présure en coagulation enzymatique, a été initialement proposé par Holter (1932) et modifié par Foltmann (1959). II s'écrit :

$$
t_{L}=K / \text { Dose }+x
$$

II est généralement appliqué à des expérimentations où le $\mathrm{pH}$ et le température sont maintenus constants. Sa principale utilisation concerne l'évaluation de l'activité coagulante des enzymes. Les résultats récents de Brown et Collinge (1986) montrent d'une part que " $X$ » n'est pas signficativement différent de 0 , et, avec les résultats de McMahon et al (1984), suggèrent d'autre part que " $X$ » est dépendant de la méthode d'observation de $t_{L}$.

Pour prendre en compte les particularités du plan d'expérience, on a testé ici le modèle:

$$
\log t_{L}=\log K+a \cdot \log (1 / \text { Dose })
$$

On trouve que $\log K$, donc $K$, dépend surtout du $\mathrm{pH}$ et à un bien moindre degré de la température, effets qui apparaissent de la même façon dans le modèle polynomial donné pour $t_{L}$. On remarque qu'en éliminant la valeur maximale observée pour le temps de latence $t_{L}=6911 \mathrm{~s}$, le coefficient "a» passe de 0,72 à 0,96 , valeur non significativement différente de 1. Les 19 observations restantes correspondent à une plage de variation qui s'étend de 425 à $5178 \mathrm{~s}$.

La valeur maximale du temps de latence (6 $911 \mathrm{~s}$ ) qui ne peut pas être ajus- tée par le modèle (7) est associée à une chute de $\mathrm{pH}$ très importante entre l'emprésurage $(p H e, 6,3)$ et la prise $\left(p H_{L} 5,6\right)$ : cette diminution du $\mathrm{pH}$ entraîne une diminution de la valeur de $t_{L}$ (fig 7) par rapport à la valeur prédite par le modèle.

L'observation $t_{L}=5178 \mathrm{~s}$, quant à elle, correspond à une variation de $\mathrm{pH}$ de 6,50 à 6,44 entre l'emprésurage et la prise.

Le modèle (7) décrit donc correctement la relation entre le temps de latence et la dose de présure en coagulation mixte, à condition que la variation de $\mathrm{pH}$ entre l'emprésurage et la prise reste limitée. Mieux ajusté que le modèle polynomial, il élimine l'artefact des temps de latence négatifs qui apparaissent avec celui-ci, sans modifier les conclusions, déduites du modèle polynomial, relatives à l'effet des facteurs $\mathrm{pH}$ et température.

La vitesse maximale de raffermissement augmente linéairement avec la Dose d'autant plus que pHe est faible, ce qui traduit une interaction significative $\mathrm{pHe}$-Dose (fig 8c, et tableau II).

Zoon et al (1988a) observent une augmentation de la vitesse de raffermissement avec la dose de présure $(0,01$ et $0,05 \%)$, alors que Bohlin et al (1984) ne mettent pas d'effet en évidence probablement par suite d'une variation très limitée de la dose de présure $(0,3-0,4 \mathrm{ml} / \mathrm{kg})$. II est admis que le pourcentage de caséinomacropeptide libéré au moment de la prise diminue lorsque la dose de présure augmente (Van Hooydonk et Van Den Berg, 1988). On peut donc penser que la vitesse de raffermissement est alors limitée par la poursuite de l'hydrolyse enzymatique de la kappa-caséine.

Si $\phi_{L}$ est indépendant de la dose de présure, ce qui confirme les résultats de Zoon et al (1988a) en coagulation enzymatique, il n'en n'est pas de même de l'angle de perte en $P_{M}$, pour lequel la Dose a un effet linéaire et quadratique (tableau II). 
Les résultats de littérature relatifs à l'effet de la dose de présure sur la "fermeté" ou le module élastique maximal, sont contradictoires. Ramet et Weber (1980) n'observent pas d'effet sur la fermeté estimée avec le Formagraph. Avec le même dispositif de mesure, mais sur une importante plage de variation de la dose de présure, les observations de McMahon et al (1984) suggèrent l'existence d'un effet quadratique sur la fermeté. Les résultats de Zoon et al (1988a) montrent un accroissement du module élastique avec la dose de présure, phénomène pour lequel les explications des auteurs sont réduites à des hypothèses.

Le sous-modèle obtenu ici pour $\tau_{M}$ montre l'existence d'un effet linéaire et quadratique de la dose de présure, mais pas d'interaction avec $\mathrm{pHe}$. La figure $8 \mathrm{a}$ traduit graphiquement ces résultats : quel que soit $p H e$, la valeur de $\tau_{M}$ augmente avec la dose jusqu'à $28-30 \mathrm{mg} / \mathrm{kg}$, puis n'évolue plus de façon significative.

Le $\mathrm{pH}$ d'emprésurage, atteint ici par l'acidification due à Streptococcus lactis diacetylactis, joue un rôle prépondérant sur l'ensemble des grandeurs caractéristiques de la cinétique de coagulation. Son effet est le plus souvent un effet linéaire, sauf pour $\phi_{L}$, et $t_{M}$ pour lesquels il a aussi un effet quadratique.

Le sous-modèle établi pour $t_{L}$ (tableau II) montre que le $\mathrm{pH}$ a un effet linéaire : lorsque $p H e$ diminue, le temps de latence diminue. En effet, le degré de protéolyse auquel survient la formation du coagulum diminue avec le $\mathrm{pH}$ (Van Hooydonk et al, 1986). Mais cette évolution est modulée par l'interaction avec la Dose (fig 8b), discutée ci-dessus.

Selon Zoon et al (1988 b), le temps de latence de la coagulation enzymatique augmente entre $\mathrm{pH} 6,75$ et 6,65 et diminue entre $\mathrm{pH} 6,65$ et $\mathrm{pH} 5,77$. Nos résul- tats correspondent à cette deuxième plage de variation du $\mathrm{pH}$.

Le $p H e$ a aussi un effet linéaire sur $V_{\text {max }}$ (tableau II, fig 6), d'autant plus fort que la Dose est forte.

L'augmentation de la vitesse de raffermissement du coagulum lorsque le $\mathrm{pH}$ diminue, entre 6,75 et 5,77 , a aussi été observée par Van Hooydonk et al (1986) et Zoon et al (1989).

Le sous-modèle qui traduit l'évolution de $\phi_{L}$ (tableau II) ne comporte que le facteur pHe : il peut être simplement représenté par une parabole dont la concavité est orientée dans le sens des valeurs positives (fig 4b). La réserve faite précédemment relative à l'approximation quadratique s'applique ici encore pour les $\mathrm{pHe}$ faibles, comme le suggèrent les points expérimentaux portés sur la figure 4b.

On peut seulement en conclure qu'aux pHe élevés, le coagulum a un comportement plus visqueux, et aux $\mathrm{pHe}$ inférieurs à 6,2 un comportement plus élastique. Enfin, les résultats présentés par Walstra et Van Vliet (1986) concernant l'évolution de $\operatorname{tg} \phi$ en fonction du $\mathrm{pH}$ devraient pouvoir être décrits en terme d'effet quadratique entre $\mathrm{pH} 5$ et $\mathrm{pH} 6$.

L'effet du pHe sur la contrainte maximale, linéaire sur l'étendue de $\mathrm{pHe}$ explorée, apparaît plus discriminant du type de coagulum : plus le $p H e$ est faible, plus $\tau_{M}$ est élevée.

D'après les résultats de Zoon et al (1989), l'évolution du module élastique maximal avec le $\mathrm{pH}$, en coagulation enzymatique, indiquerait un effet de même sens entre $\mathrm{pH} 6,6$ et 6,2 mais une diminution entre 6,2 et 5,7 .

Le temps caractéristique $t_{M}$ du point $P_{M}$ décroît avec le $p H e$ jusqu'à 6,2 . En effet, la position de $P_{M}$ résulte de la superposition de 2 courbes d'évolution de la "fermeté " : 
d'une part une augmentation due au phénomène de coagulation, d'autre part une diminution due à la déminéralisation des micelles liées à l'acidification. Par suite, plus le $\mathrm{pHe}$ sera bas et plus ces courbes seront décalées vers les temps courts après l'emprésurage et donc plus $t_{M}$ sera petit. L'absence d'interaction Dose-pHe sur $t_{M}$ s'explique de la façon suivante: la Dose n'agit pas sur la cinétique d'acidification donc sur la déminéralisation et enfin, les effets d'interaction trouvés sur le temps de latence d'une part, et sur $V_{\max }$ d'autre part se compensent.

Nous venons de discuter les résultats d'un plan d'expérience au moyen d'un ajustement de modèles aux données expérimentales. II convient, avant de conclure, de préciser quelques aspects relatifs aux ajustements. Une erreur pure très petite signifie une bonne maîtrise des expérimentations, ce qui est le cas ici, et par suite fait ressortir plus nettement un défaut d'ajustement. Un défaut d'ajustement grand en valeur ne signifie pas que le modèle est une mauvaise représentation de la réalité; les effets détectés peuvent néanmoins constituer une très bonne indication de la hiérarchie des facteurs. L'examen des résidus (tableau V) complète l'appréciation de la qualité de l'ajustement.

\section{CONCLUSION}

II est indéniable que beaucoup des résultats présentés ici confirment un savoir existant : ils contribuent cependant à quantifier les différents effets et leur importance relative. De plus, ils présentent un aspect original par la mise en évidence et la quantification d'effets "quadratiques» et d'interactions. Certains résultats de la littérature suggèrent, de toute évidence, l'existence d'effets quadratiques, mais ceux-ci ne sont jamais analysés explicitement.
L'influence de la température est généralement étudiée sur une large étendue, alors qu'en pratique, pour un type de fabrication, l'intérêt de connaître l'effet de la température concerne une plage faible. On montre ici que les effets sont limités sinon insignifiants pendant les premières phases de la coagulation mixte; ce n'est qu'aux temps longs que les conséquences apparaissent nettement, surtout par l'accentuation des phénomènes de synérèse. Mais en technologie fromagère, les étapes qui suivent la fabrication sont mises en œuvre bien avant la manifestation de ces phénomènes.

Déjà relativement bien connus, les rôles du $\mathrm{pH}$ à l'emprésurage et de la dose de présure sont ici quantifiés et précisés, notamment par l'interaction qu'ils exercent sur certaines grandeurs caractéristiques de la coagulation mixte. On montre que des combinaisons différentes du $\mathrm{pHe}$ et de la Dose peuvent conduire à une même valeur du temps de latence, mais des évolutions très différentes du coagulum en terme de raffermissement. Le modèle classique entre le temps de latence et l'inverse de la dose de présure, utilisé fréquemment en coagulation enzymatique, s'applique en coagulation mixte dans certaines limites qui sont précisées.

Le traitement du plan d'expérience présenté ici illustre enfin l'utilisation des polynômes orthogonaux en régression polynomiale, qui permettent d'interpréter et de comparer entre eux les effets des facteurs étudiés. L'intérêt et les limites de cette technique d'analyse sont discutés.

\section{REMERCIEMENTS}

Nous adressons nos plus vifs remerciements à R Grappin, directeur de la Station de recherches en technologie et analyses laitières (INRA Poligny) et $J$ Lefèbvre, directeur du Laboratoire de 
physico-chimie des macromolécules (INRA Nantes) pour leurs conseils et leurs remarques pertinentes lors de la rédaction de cet article.

Nous remercions également A Dasen (INRA Poligny) pour sa contribution déterminante quant au choix de la souche de bactérie lactique, ainsi que $S$ Alige (INRA Massy) pour sa collaboration technique dans la préparation des ferments lactiques.

\section{RÉFÉRENCES}

Bohlin L, Hegg PO, Ljusberg-Waren H (1984) Viscoelastic properties of coagulating milk. $J$ Dairy Sci 67, 729-734

Box GEP, Wilson KB (1951) On the experimental attainment of optimum conditions. $J R$ Stat Soc B 13, 1-45

Box GEP, Hunter JS (1957) Multifactor experimental designs for exploring response surfaces. Ann Math Stat 28, 195-241

Brown RJ, Collinge SK (1986) Actual milk coagulation time and inverse of chymosin activity. J Dairy Sci 69, 956-958

Cheryan M, Van Wyk PJ, Olson NF, Richardson $T$ (1975) Secondary phase and mechanism of enzymic coagulation. J Dairy Sci 58,477 481

Collin JC, Grappin R, Le Graet Y (1977) Étude de la méthode de mesure, selon Berridge, du temps de coagulation du lait additionné d'une solution enzymatique. Rev Lait Fr 355 , 389-394

Dejmek P (1987) Dynamic rheology of rennet curd. J Dairy Sci 70, 1325-1330

Durier C, Kobilinsky A (1989) APOLOR : une nouvelle vision de la régression polynomiale. XXle Journ Stat, Rennes

Eck A (1984) Le Fromage, Lavoisier, Paris

Famelart MH, Maubois JL (1988) Comparaison de l'indice de réfraction et de la viscosité au cours de la gélification du lait. Lait 68, 1-12

FIL (1987) Détermination of chymosin and bovine pepsin contents. Norme FIL 110A

Foltmann B (1959) On the enzymatic and coagulation stages of the renneting process. 15th Int Dairy Congr Londres 2, 655

Gervais A, Vermeire D, Cerf O, Toux J (1982) Procédé et appareil de mesure des propriétés rhéologiques de corps semi-solides par cisaillement harmonique en rotation. Demande de brevet français $n^{\circ} 82.17835$
Harwalkar VR, Kalab M (1980) Milk gel structure. XI. Electron microscopy of gluconodelta-lactone-induced skim milk gels. $J$ Texture Stud 11, 35-49

Harwalkar VR, Kalab M (1981) Effects of acidulants and temperature on microstructure, firmness and susceptibility to syneresis of skim milk gels. Scann Electron Microsc 3, 503-513

Heertje I, Visser J, Smits P (1985) Structure formation in acid milk gels. Food Microsc 4, 267-277

Holter H (1932) Uber die Labwirkung. Biochem $Z 255,160$

John PWM (1971) Statistical design and analysis of experiments. Macmillan, Londres

Khuri Al, Cornell JA (1987) Response surfaces. (M Dekker, ed), New-York

Kim BY, Kinsella JE (1989) Effect of temperature and $\mathrm{pH}$ on the coagulation of casein. Milchwissenschaft 20, 1-10

Kobilinsky A (1988) Tactiques en analyse de variance et en régression. Rev Modulad 1, 2558

Kowalchyk AW, Olson NF (1977) Effects of pH and temperature on the secondary phase of milk clotting by rennet. $J$ Dairy Sci $67,263-$ 269

Lacroix C, Castaigne F (1986) Application des plans expérimentaux factoriels fractionnés et de la technique de surface de réponse à l'optimisation d'un produit ou d'un procédé dans le domaine alimentaire. Ind Alim Agric 103, 105-113

Lee KT (1986) Contribution à l'étude cinétique de la coagulation du lait par la présure : comparaison de deux méthodes rhéologiques. Thèse Univ Paris VII, Paris XI, ENSIA

Mc Mahon DJ, Brown RJ (1984) Enzymatic coagulation of casein micelles: a review. J Dairy Sci $67,1185-1193$

Myers RH (1971) Response surface methodology. Allyn and Bacon Inc, Boston

Noël Y (1989) Comparaison des cinétiques de coagulation enzymatique et mixte du lait. Influence du calcium. Lait 69, 479-490

Phan Tan Luu R, Feneuille D, Mathieu D (1983) Méthodologie de la recherche expérimentale. Étude des surfaces de réponse publiée par le laboratoire de prospective réactionnelle et d'analyse de l'information, IPSOI, Marseille

Ramet JP, Weber F (1980) Contribution à l'étude de l'influence des facteurs de milieu 
sur la coagulation enzymatique du lait reconstitué. Lait 60, 1-13

Roefs SPFM (1985) Structure of acid casein gels. A study of gels formed after acidification in the cold. Ph D Thesis, Agric Univ Wageningen, Netherlands

Shalabi SI, Fox PF (1982) Influence of pH on the rennet coagulation of milk. I Dairy Res $49,153-157$

Tokita M, Futakuchi H, Hikichi K, Niki R, Arima S (1982) Dynamic viscoelastic studies on the mechanism of milk clotting process of casein micelle solution. Biorheology 19, 209-219

Tokita M, Futakuchi H, Hikichi, Niki R, Arima S (1983) Dynamic mechanical properties of milk and milk gel. Biorheology 20, 1-10

Van den Bijgaart HJCM (1988) Syneresis of rennet-induced milk gels as influenced by cheesemaking parameters. Ph D Thesis Agric Univ Wageningen, The Netherlands

Van Hooydonk ACM, Van den Berg G (1988) Control and determination of the curd-setting during cheesemaking. Bull FIL 225

Van Hooydonk ACM, Boerrigter IJ, Hagedoorn $H G$ (1986) $\mathrm{pH}$ induced physico-chemical changes of casein micelles in milk and their effect on renneting. 2. Effect of $\mathrm{pH}$ on renneting of milk. Neth Milk Dairy J 40, 297-313

Van Vliet T, Roefs SPFM, Zoon P, Walstra P (1989) Rheological properties of casein gels. J Dairy Res 56, 529-534

Walstra P, Jenness R (1984) Dairy chemistry and physics. Wiley Intersci publ, J Wiley \& sons, New York

Walstra P, Van Vliet T (1986) The physical chemistry of curd making. Neth Milk Dairy $J 40$, 241-259

Walstra P, Van Dijk HJM, Geurts TJ (1985) The syneresis of curd. 1. General considerations and literature review. Neth Milk Dairy J 39, 209-246

Zoon P, Van Vliet T, Walstra T (1988a) Rheological properties of rennet-induced skim milk gels. 1. Introduction. Neth Milk Dairy J 42, 249-269

Zoon P, Van Vliet T, Walstra T (1988b) Rheological properties of rennet-induced skim milk gels. 2. The effect of temperature. Neth Milk Dairy J 42, 271-294

Zoon P, Van Vliet T, Walstra T (1989) Rheological properties of rennet-induced skim milk gels. 4. The effect of $\mathrm{pH}$ and $\mathrm{NaCl}$. Neth Milk Dairy J 43, 17-34 


\section{ANNEXE : ANALYSE DE $t_{L}$ (TEMPS DE LATENCE)}

L'expérimentation a été conduite en 20 essais de manière à estimer dans un modèle de degré 2 , les effets moyens, linéaires, quadratiques et les interactions lin.lin des 3 facteurs étudiés. Ces effets sont les coefficients des polynômes orthonormés $P_{0}, P_{1}, P_{2}$ dans le modèle :

$\mathrm{t}_{\mathrm{L}}=a_{0}^{\prime} P_{0}+b_{1}^{\prime} P_{1}(T)+b_{2}^{\prime} P_{2}(T)+c_{1}^{\prime} P_{1}(p H e)+c_{2}^{\prime} P_{2}(p H e)+d_{1}^{\prime} P_{1}($ Dose $)$

$+d_{2}^{\prime} P_{2}($ Dose $)+e^{\prime} P_{1}(T) P_{1}(p H e)+f^{\prime} P_{1}(T) P_{1}($ Dose $)+g^{\prime} P_{1}(p H e) P_{1}($ Dose $)$

(Kobilinsky, 1988; Durier et Kobilinsky, 1989) où $T$, Dose, pHe sont les facteurs codés. $P_{0}, P_{1}, P_{2}$ sont définis sur le plan complet ( $5 \times 5 \times 5$ combinaisons) et ils sont obtenus en normant les polynômes orthogonaux $P_{0}^{\prime}, P_{1}^{\prime}, P_{2}^{\prime}$ qui sont, par exemple pour $T$ :

$$
\begin{array}{rll}
P_{0}^{\prime} & = & 1 \\
P_{1}^{\prime}(T) & = & T \\
P_{2}^{\prime}(T) & = & T^{2}-m_{2} \\
\text { où } m_{2}=1 / 5 & \times\left((-1,6)^{2}+(-1)^{2}+(0)^{2}+(1)^{2}+(1,6)^{2}\right)=1,424
\end{array}
$$

Cette normation, qui les ramène à des échelles de variation comparables, est effectuée en les divisant par leurs écarts types qui sont :

$$
\begin{array}{ll}
\text { écart type }\left(P^{\prime}, 0\right) & =1 \\
\text { écart type }\left(P^{\prime},(T)\right)= & \sqrt{m_{2}} \\
\text { écart type }\left(P_{2}^{\prime}(T)\right)= & \sqrt{\left(m_{4}-m_{2}{ }^{2}\right)} \\
\text { où } m_{4}=1 / 5 \times\left((-1,6)^{4}+(-1)^{4}+(0)^{4}+(1)^{4}+(1,6)^{4}\right)=3,021
\end{array}
$$

Les polynômes orthogonaux normés obtenus sont donc :

$$
P_{0}=1 \quad P_{1}(T)=\frac{T}{1,193} \quad P_{2}(T) \quad \frac{T^{2}-1,424}{0,997}
$$

et de même pour les autres facteurs pHe et Dose.

Les coefficients $a_{0}^{\prime}, b_{1}^{\prime}, \ldots, g^{\prime}$ ou effets sont estimés par la méthode des moindres carrés. Le tableau A-I présente les estimations des effets (équation 4) en première colonne, puis les intervalles de confiance à différents seuils $(90,95$ et $99 \%$ ) (colonnes 2,3 et 4 ) et enfin, les estimations des coefficients du modèle polynômial original (équation 3). Ce tableau permet de sélectionner un sous-modèle englobant tous les effets significatifs à $95 \%$ (seuil pour lequel on retient les effets importants) repérés par un astérisque.

On constate dans ce cas que les effets éliminés sont nettement plus faibles que les effets retenus, on peut donc conclure que $t_{L}$ dépend essentiellement de $\mathrm{pHe}$, Dose, Dose ${ }^{2}, \mathrm{pHe}$. Dose.

Le modèle ainsi retenu est :

$$
\begin{aligned}
t_{L} & =2505,9+998,1 \frac{p H e}{1,193}-1780,5 \frac{\text { Dose }}{1,193}-792,5 \frac{p H e}{1,193} \cdot \frac{\text { Dose }}{1,193}+975,9 \frac{\text { Dose }^{2}-1,424}{0,997} \\
& =1112,9+836,4 p H e-1492 \text { Dose }-556,5 \text { pHe.Dose }+978,4 \text { Dose }^{2}
\end{aligned}
$$

Ce modèle, donnant une représentation simplifiée des phénomènes conduit au graphique en 3 dimensions (fig 4a) qui facilite l'interprétation. Les modèles obtenus pour les autres grandeurs caractéristiques font l'objet de figures analogues (fig $4 b, 4 c, 5 a, 5 b, 5 c, 5 d$ et 6 ). 
Tableau A-I

$t_{L}$ : Estimations des effets, intervalles de confiance et coefficients

\begin{tabular}{lrrrrr}
\hline & Effet & + - seuil90 & seuil95 & seuil99 & coeff monôme \\
\hline moy & 2505,9 & 462,7 & 568,8 & 809,1 & 1112,9 \\
* lin T & $-160,9$ & 335,2 & 412,1 & 586,2 & $-134,9$ \\
* lin Dose & 998,1 & 335,2 & 412,1 & 586,2 & 836,4 \\
lin quad T & $-1780,5$ & 335,2 & 412,1 & 586,2 & $-1492,0$ \\
lin T.lin pHe & 19,8 & 287,6 & 353,6 & 502,9 & 19,8 \\
lin T.lin Dose & $-141,7$ & 512,3 & 629,8 & 895,8 & $-99,5$ \\
quad pHe & 146,7 & 512,3 & 629,8 & 895,8 & 103,0 \\
* lin pHe.lin Dose & 164,4 & 287,6 & 353,6 & 502,9 & 164,9 \\
quad Dose & $-792,5$ & 512,3 & 629,8 & 895,8 & $-556,5$ \\
& 975,9 & 287,6 & 353,6 & 502,9 & 978,4 \\
\hline
\end{tabular}

Un effet est signicatif par exemple à $95 \%$ si [Effet \pm seuil95] ne contient pas 0; Écart type résiduel $=561,4$ (10 degrés de liberté).

\section{Estimation de l'effet "Température" sur $\mathrm{t}_{L}$ pour une variation de $2{ }^{\circ} \mathrm{C}$}

Bien que non significative, la variation de $t_{L}$ induite par celle de $T$ entre $32^{\circ} \mathrm{C}$ et $34^{\circ} \mathrm{C}$ peut être évaluée comme suit :

l'estimation de l'effet linT est $-160,9$ (tableau A-I).

Pour le codage adopté ici, la variation de $t_{L}$ pour $1^{\circ} \mathrm{C}$ est donc estimée à $-160,9 / 1,193$ et pour $2^{\circ} \mathrm{C}$ à $2 \times-160,9 / 1,193=-270$.

Ceci étant rapporté à la moyenne générale (moy $=2505,9)$, on peut estimer grâce à cette expérience une diminution de $t_{L}$ de $11 \%$ pour $T$ passant de $32^{\circ} \mathrm{C}$ à $34^{\circ} \mathrm{C}$. 